\title{
Numerical modelling of the effects of consolidation on jack-up spudcan penetration.
}

Manuscript submitted to Computers and Geotechnics on 14/12/2015 by:

Raffaele Ragni ${ }^{1}$ (corresponding author)

PhD candidate

Tel: +61 (0) 864887254

Email:21358454@student.uwa.edu.au

Dong Wang ${ }^{1}$

Senior Research Fellow

Tel: +61 (0) 864883469

Email: dong.wang@uwa.edu.au

David Mašín ${ }^{2}$

Associate Professor

Tel: +42022195 1552

Email: masin@natur.cuni.cz

Britta Bienen ${ }^{1}$

Senior Lecturer

Tel: +61 (0) 864884246

Email: britta.bienen@uwa.edu.au

Mark J. Cassidy ${ }^{1}$

Professor

Director

Tel: +61 (0) 864883732

Email: mark.cassidy@uwa.edu.au

Samuel A. Stanier ${ }^{1}$

Research Fellow

Tel: +61 (0) 864883469

Email: sam.stanier@uwa.edu.au

${ }^{1}$ Centre for Offshore Foundation Systems and ARC CoE for Geotechnical Science and Engineering

University of Western Australia

35 Stirling Hwy

Crawley, Perth, WA 6009

Australia

${ }^{2}$ Faculty of Science

Charles University in Prague

Albertov 3, 12843 Praha

Czech Republic 


\section{ABSTRACT}

The paper addresses the issue of consolidation around jack-up foundations in carbonate silty clay. The problem is tackled with the numerical implementation of a hypoplastic model for structured clays, within the framework of large deformation finite element analyses. Coupled analyses are simulated to account for excess pore pressure build-up and dissipation, while sensitivity parameters capture the effects of remoulding. The model implementation is described first, followed by its validation against centrifuge data. The paper concludes with a detailed discussion of beneficial or detrimental effects on spudcan capacity upon re-penetration, depending on the consolidation and soil sensitivity characteristics.

Keywords: Spudcan; Consolidation; Bearing capacity; Numerical modelling; Hypoplasticity; Sensitivity. 


\section{INTRODUCTION}

Installation of an offshore jack-up platform typically involves the penetration of circular footings - also known as spudcans - into the soil under the self-weight of the structure, followed by progressively pumping sea water into the ballast tasks until the target pre-load is reached. Although it is desirable for this procedure to be conducted as quickly as possible, interruptions may be experienced during installation, causing the spudcan to sit at a certain depth for a period of time (see Figure 1 which provides a schematic illustration of pre-load and penetration time history). During these pauses in penetration some consolidation can take place in the soil around the spudcan, with a consequent increase in soil shear strength and stiffness. This can set the conditions for a 'consolidation-generated' punch-through, where the spudcan quickly advances through the strengthened zone when penetration restarts. This is recognised in ISO (2012), though no further advice of how to predict it - or mitigate it - is offered. Episodes of field failure have been reported: Brennan et al. (2006) showed that even short pauses in the order of hours contributed to the subsequent punch-through failure. Consolidation can also be actively sought in order to limit spudcan penetration, as detailed in Erbrich (2005). In either scenario, comprehensive understanding of the physical process occurring, and its effects on the geotechnical behaviour, is critical to formulate a detailed installation plan and to mitigate risk.

To date, the relevant data base is scarce. Barbosa-Cruz (2007) and Bienen \& Cassidy (2013) carried out a small number of centrifuge experiments on spudcan penetration-consolidation-penetration cycles, with the latter highlighting the punch-through risk created through pauses in footing penetration in kaolin clay. In a natural soil recovered from offshore Australia (a carbonate silty clay) further centrifuge experiments revealed the potential for a beneficial increase in bearing capacity due to consolidation without the elevation of the risk of punch-through (Bienen et al 2015). The mechanisms of jack-up 
foundation penetration pre- and post-consolidation were seen to differ in Particle Image Velocimetry (PIV) experiments, as reported in Stanier et al. (2014). Comparable peaks in bearing capacity post-consolidation were observed for kaolin clay and carbonate silty clay in all of the experiments reported. However, it has not been possible to isolate the effects of the changing failure mechanism and local changes in undrained shear strength and stiffness from the PIV analyses. In addition, centrifuge modelling, especially on natural soil, is not economic for extensive parametric studies.

Numerical modelling can offer the desired insights provided a large deformation approach is used that features coupled pore fluid-stress response and a constitutive model that captures salient features of soil behaviour. This is necessary to model the process of installation of a spudcan footing which involves deep penetration, which can be up to three diameters. The numerical model further needs to account for consolidation. A coupled effective stress-pore fluid analysis is thus required, such that excess pore pressures generated during penetration can dissipate leading to increases in the effective stresses in the soil.

Natural offshore soils can be highly sensitive (with sensitivities up to 20 in the carbonate deposits found offshore Australia; Erbrich 2005), a constitutive model of the hypoplasticity family previously developed for structured clays (Mašín 2007) was incorporated in the numerical analyses of this study. The model has the ability to capture the incrementally non-linear behaviour of the soil as well as the effects of remoulding and softening due to soil sensitivity. As this is the first time this constitutive model has been used in a complex boundary value problem like this, the paper presents both model background and validation in detail. Then, the capabilities of the hypoplastic model are harnessed, which allow aspects of soil response such as the effect of high soil sensitivity to be explored that cannot be captured using Modified Cam Clay, for instance. A detailed investigation of the increase in bearing capacity is presented, through a wide parametric study on the effects of consolidation 
length, depth and load-hold, as well as the sensitivity properties. In terms of length of consolidation, the study aims to cover different scenarios, from short pauses during installation to longer vertical load-hold periods (i.e. self-weight of the structure). On one side, short pauses in penetration - in the order of hours - can be seen either as beneficial for the jack-up in order to reduce the spudcan installation depth or as a detrimental instigator for punch-through. On the other side, longer periods of consolidation - in the order of months or even years - will not occur during installation, but can enhance the bearing capacity over time. For this reason, the enhancement in bearing capacity has to be seen as potentially beneficial relative to the operational conditions (by improving the fixity of the foundations). Finally, the study on sensitivity parameters illustrates the different behaviours observed when natural, rather than reconstituted soil is modelled, quantifying first the increase in bearing capacity due to consolidation, and then its reliability upon re-penetration, showing once again the possibility of either beneficial or disadvantageous responses.

\section{NUMERICAL MODEL}

\subsection{Large deformation approach with coupled pore fluid-stress response}

The large deformation finite element approach adopted in this paper is based on the Remeshing and Interpolation Technique with Small Strain (RITSS) strategy (Hu \& Randolph 1998). The RITSS technique has been extended to model coupled pore fluid-stress response in the commercial code Abaqus/Standard (Dassault Systemes 2012) as described in Wang et al. (2008). The basic premise of RITSS large deformation finite element analysis is to divide the entire analysis into small incremental steps so that mesh distortion is minimised and consecutive finite strain analyses are executed. A new mesh is generated at the beginning of every step based on the final deformed configuration of the previous one. Then the effective stresses and state variables are mapped to each integration point and the pore pressures to element nodes, with convection achieved by polynomial interpolation. Irrespective of 
whether the variables are mapped to the new integration points or to the new nodes, the interpolation in RITSS is always conducted locally within an old element, an old element patch or a triangle connecting old integration points (Wang et al. 2015). In this study, a modified unique element method proposed by Hu \& Randolph (1998) was employed to map effective stresses and material state properties from the old integration points to the new integration points.

\subsection{Geometry, mesh and boundary conditions}

The numerical analyses were conducted using axisymmetric boundary conditions since most spudcan foundations are circular. The spudcan was modelled as a rigid body due to its much higher stiffness compared to the soil. The interaction at the soil-spudcan interface was simplified as frictionless as the interface roughness, even in consolidation problems, has been shown to have little influence on the resulting spudcan penetration resistance (Wang \& Bienen 2015). Eight-node elements with biquadratic displacement, bilinear pore pressure and reduced integration (termed CAX8RP in Dassault Systemes 2012) were used to discretise the soil.

The soil domain was extended $20 \mathrm{D}$ (where $D$ is the spudcan diameter) both vertically and horizontally to avoid boundary effects, modelling a large expanse of single layer soil in the field. The minimum element size around the spudcan was $0.025 \mathrm{D}$ and it had minimal effect on the simulated penetration resistance when reduced to $0.015 \mathrm{D}$. The numerical model is shown in Figure 2, where vertical and horizontal degrees of freedom were constrained for the bottom of the region and the two vertical sides respectively. Drainage of excess pore pressures was allowed through the soil surface. The vertical spudcan displacement for each incremental step of the RITSS analyses was taken as $0.01 \mathrm{D}$, small enough to avoid excessive mesh distortion, after which the mesh was periodically regenerated. 
The numerical analyses were performed at model scale rather than at prototype dimension, in order to make a straightforward comparison with the centrifuge tests that are discussed in the validation section. Penetration of the spudcan from the soil surface is challenging to perform numerically especially for normally consolidated soil profiles, as heaving soil around the foundations changes geometry suddenly upon failure caused by its self-weight; hence an initial embedment of the spudcan of $0.5 \mathrm{D}$ was prescribed to ease convergence.

\subsection{Hypoplastic constitutive model for structured clays}

\subsubsection{Model description}

Numerous studies (Atkinson 2000, Gasparre 2005) have demonstrated the reduction in soil stiffness from very small to very large strain and the incrementally non-linear behaviour of soil. Such behaviour cannot be modelled using conventional elasto-plastic constitutive models such as Modified Cam Clay, where a yield surface in the stress space bounds the admissible states and the behaviour inside it is reversible. It is instead necessary to introduce irreversible strains inside the state boundary surface, as in soil behaviour the elastic range is extremely limited. Therefore, the choice to adopt a hypoplastic model is a step beyond the conventional elasto-plasticity.

Hypoplasticity models have been developed to describe the behaviours of clays and sands. In its general form (Gudehus 1996) it may be written as

$$
\dot{\boldsymbol{\sigma}}=f_{s}\left(\boldsymbol{L}: \dot{\boldsymbol{\varepsilon}}+f_{d} \boldsymbol{N}\|\dot{\boldsymbol{\varepsilon}}\|\right)
$$

where $\dot{\sigma}$ and $\dot{\varepsilon}$ represent the objective stress rate and the Euler stretching tensor, respectively, $\boldsymbol{L}$ and $\boldsymbol{N}$ are the fourth- and second-order constitutive tensors, and $f_{s}$ and $f_{d}$ are two scalar factors. In hypoplasticity, the stiffness predicted by the model is controlled by tensor $\boldsymbol{L}$, 
whereas the strength (and asymptotic response in general) is governed by a combination of $\boldsymbol{L}$ and $\boldsymbol{N}$. Earlier hypoplastic models, such as those of Von Wolffersdorff (1996) and Mašín (2005), did not allow arbitrarily changing of the $\boldsymbol{L}$ formulation as any modification of tensor $\boldsymbol{L}$ undesirably influenced the predicted asymptotic states. This hypoplasticity limitation was overcome by Mašín (2012) who developed an approach enabling the specification of the asymptotic state boundary surface independently from tensor $\boldsymbol{L}$. The efficacy of this approach was demonstrated by proposing a simple hypoplastic equivalent of the Modified Cam-clay model. Based on this approach, Mašín (2014) developed an advanced hypoplastic model for clays, which is adopted in the present work as an updated version of the Abaqus/Standard user subroutine (Dassault Systemes 2012) by Gudehus et al. (2008).

The basic hypoplastic clay model requires five material properties $\varphi_{c}, N, \lambda^{*}, \kappa^{*}$ and $v$. The parameters have the similar physical interpretation as the parameters of the Modified Cam clay model, and they are thus easy to calibrate using standard laboratory experiment. The model parameters $N$ and $\lambda^{*}$ define the position and the slope of the isotropic normal compression line in the $\ln (1+e) \sim \ln \left(p^{\prime} / p_{r}\right)$ plane. The isotropic normal compression line is described using the equation

$$
\ln (1+e)=N-\lambda^{*} \ln \left(\frac{p^{\prime}}{p_{r}}\right)
$$

where $p_{r}=1 \mathrm{kPa}$ is a reference stress, $e$ the void ratio and $p^{\prime}$ the mean effective stress. Parameter $\kappa^{*}$ controls the slope of the isotropic unloading line in the same plane and also of the isotropic compression line of overconsolidated soil. $\varphi_{c}$ is the critical state friction angle, with identical meaning to that in any other critical state soil mechanics-based model. Finally, the parameter $v$ controls the shear modulus and reads: 


$$
v=\frac{3 r\left(\lambda^{*}+\kappa^{*}\right)-4 \kappa^{*}}{6 r\left(\lambda^{*}+\kappa^{*}\right)+4 \kappa^{*}}
$$

where $r$ is the ratio of the bulk modulus in isotropic compression at the isotropic normally consolidated state and the shear modulus in undrained shear.

Apart from the stress, the most important state variable controlling the response of the model is the void ratio $e$. In the numerical simulation, this parameter can be directly initialised as a constant. However, such an approach has a significant drawback as the degree of overconsolidation with depth is increased. In this case, the void ratio is initialised in an alternative manner, ensuring a constant over-consolidation ratio OCR with depth, consistently with the conditions found in a normally consolidated deposit. The OCR in the hypoplastic model is defined as

$$
\text { OCR }=\frac{p_{e}}{p^{\prime}}
$$

where $p_{e}$ is the Hvorslev equivalent pressure, that is, the mean effective stress at the isotropic normal compression line calculated using Equation (2) at the current void ratio. Note that this definition of $O C R$ is slightly different from the traditional soil mechanics definition, where OCR is a ratio of pre-consolidation pressure and mean stress. It is also important to point out that in cases like the present work, where the soil is in normally consolidated (i.e. oedometric) conditions, $O C R$ is not equal to 1 , as the oedometric normal compression line is below the isotropic normal compression line in the $\ln (1+e) \sim \ln \left(p^{\prime} / p_{r}\right)$ plane. To initialize the state for $K_{0}$ consolidated conditions, with the earth pressure coefficient at rest $K_{0}=1-\sin \left(\varphi_{c}\right)$ (assuming a constant $\varphi_{c}$ after triaxial simulations of Figure 6) governing the ratio of 
horizontal over vertical stresses (Rott et al. 2015), a value of $\operatorname{OCR}\left(K_{0}\right)$ must be calculated from the model equations.

The basic hypoplastic model was also enhanced by the effects of sensitivity to capture the degree of remoulding in the soil, using a procedure developed in Mašín (2007). This enhancement requires three additional parameters, $k, A$ and $s_{f}$, and the sensitivity $s$ as a state variable. Sensitivity $s$ is here defined in the way as proposed by Cotecchia \& Chandler (2000): it is representative of the ratio of both the undrained shear strengths and the ratio of pre-consolidation pressures for undisturbed and reconstituted soils. The parameter $k$ controls the rate of the sensitivity degradation

$$
\dot{s}=-\frac{k}{\lambda^{*}}\left(s_{i n i}-s_{f}\right) \dot{\varepsilon}^{d}
$$

where $s_{i n i}$ and $s_{f}$ represent the initial and final values of sensitivity, and $\dot{\varepsilon}^{d}$ is the damage strain rate. The parameter $A$, which only has a minor effect on the results, controls the relative importance of the volumetric strain $\varepsilon^{v}$ and shear strain $\varepsilon^{s}$ components

$$
\dot{\varepsilon}^{d}=\sqrt{\left(\dot{\varepsilon}^{v}\right)^{2}+\frac{A}{1-A}\left(\dot{\varepsilon}^{s}\right)^{2}}
$$

The normal compression line shown in Figure 3 then reads

$$
\ln (1+e)=N-\lambda^{*} \ln \left(\frac{p}{p_{r}}\right)+\lambda^{*} \ln (s)
$$

and the over-consolidation ratio is defined using $O C R=p_{e} / p^{\prime}=s p_{e}{ }^{*} / p$, where $p_{e}$ is the Hvorslev equivalent pressure on the isotropic normal compression line of the structured soil and $p_{e}{ }^{*}$ is the same equivalent pressure of the reconstituted soil. 
The clay hypoplastic model, in its default form, assumes fixed relative position of critical state line in the $\ln (1+e) \sim \ln \left(p^{\prime} / p_{r}\right)$ plane with respect to the isotropic normal compression line. For the given void ratio, the pressure on the critical state line $p_{c}$ is equal to $p_{e} / 2$. This ratio is consistent with the Modified Cam-clay formulation and it is suitable for most standard soils. However, the carbonate silty clay investigated here is non-standard and it has been found that the relative positions of the critical state and isotropic normal compression line are different from the default values. The model formulation has for this reason been enhanced by a parameter $O_{c}$, which controls the position of the critical state line in the following way

$$
p_{c}=\frac{p_{e}}{O_{c}}
$$

With $O_{c}=2$ the default model is recovered. The parameter $O_{c}$ affects the shape of the state boundary surface, and consequently, also the undrained stress paths, undrained stress-strain curves and undrained shear strength. Figures 4a,b show the shape of the Asymptotic State Boundary Surface (ASBS) and undrained stress paths and stress-strain curves of $K_{0}$ normally consolidated Laminaria soil. From the shape of ASBS and the definition of sensitivity the undrained shear strength corresponding to the given degree of sensitivity s predicted by the hypoplastic model is given by

$$
s_{u}=\frac{O C R p^{\prime} M}{2 O_{c}} s
$$

where $\mathrm{M}$ is the slope of critical state line in the $\mathrm{q} \sim \mathrm{p}$ plane, which can be calculated from $\mathrm{M}=6 \sin \varphi_{c} /\left(3-\sin \varphi_{c}\right)$ for triaxial compression. Although the model does not explicitly feature a residual state friction angle, the critical state strength and current OCR do not imply a lower bound to the undrained strength of the soil. This is because the model considers 
sensitivity as a state variable: soil reaches critical state stress ratio with sensitivity larger than one, i.e. in natural conditions. Further shearing and consequent strain increase causes sensitivity to decrease and the soil to reach a fully remoulded residual state, represented by sensitivity equal to one.

\subsubsection{Parameter calibration}

The soil used in the experiments is a carbonate silty clay recovered from the Laminaria field, in the Timor Sea off the Northern Coast of Western Australia. It has a particle distribution consisting of about $10 \%$ sand, $70 \%$ silt and $20 \%$ clay and a specific unit weight expressed in $\mathrm{kN} / \mathrm{m}^{3}$ as a function of the depth $w$ in $\mathrm{m}: \gamma^{\prime}=5.6+0.022 w$ (Randolph et al. 1998). The elevated calcium-carbonate content up to $85 \%$ and grain crushing at increasing stress level give this soil characteristic of 'clay-like' elevated plasticity (Erbrich \& Hefer 2002). Further studies on similar carbonate soils also revealed this silt to be a 'difficult' and 'challenging' soil (Erbrich 2005, Amodio et al. 2015).

The basic material properties of the Laminaria soil were evaluated through standard laboratory tests. Throughout the paper, 'Exp' refers to experimental results, 'Num' to numerical results. In particular, $N, \lambda^{*}$ and $\kappa^{*}$ were fitted from experimental oedometer test results of Figure 5, carried out starting from soil in a fully remoulded state, i.e. with $s_{i n i}=1$. Triaxial tests on $K_{0}$ consolidated samples (Figure 6) revealed a slope $M=1.375$ when results are plotted in the $q \sim p^{\prime}$ plane and a consequent $\varphi_{c}=34$ (Figure 6b). $v=0.1$ was identified as appropriate through numerical parametric study of triaxial tests. Given the observation of shear bands in the samples and the inability of the model to capture this phenomenon, the response of the simulations is quite different to the experimental response (this discrepancy is revisited in a later discussion at the end of this section). However, the slope of the critical state line $M$ was nonetheless captured. 
Back-analysis of the results from the centrifuge tests of Bienen et al. (2015) underpinned the calibration of the remaining parameters. A series of experiments involving full spudcan penetrating into carbonate silty clay was carried out in a beam centrifuge at the University of Western Australia (UWA) at enhanced gravity level of $a=200 \mathrm{~g}$. Before starting the testing program, the undrained soil shear strength was evaluated as $s_{u}=2.2 \mathrm{w} \mathrm{kPa}$ using a miniature piezoball penetrometer, assuming a factor $N_{\text {Ball }}=10.5$. The spudcan had a diameter $D=60 \mathrm{~mm}$ and was equipped with five pressure transducers, three on the bottom surface and two on the top surface, in order to monitor the pore pressure variations.

Cyclic T-bar tests, reported in Figure 7, showed an average value of sensitivity $s=s_{u} / s_{u, i n i}=2.9$ for the carbonate silty clay, where $s_{u, \text { ini }}$ is the initial, intact undrained shear strength of the sample and $s_{u}$ the remoulded value. Although these centrifuge tests were carried out on real soil recovered from the Laminaria field, the sample used for centrifuge tests in Bienen et al. (2015) was reconsolidated under its self-weight only. For this reason it is very likely that part of the original natural sensitivity - intended by Baudet \& Stallebrass (2004) as a combination of fabric and bonding - was lost during sampling and reconstitution. In particular, the high degree of disturbance due to sampling would erase the bonding forces between soil particles, which are achieved with extremely long periods of sedimentation and diagenesis, whereas at least part of the fabric can be recovered during centrifuge consolidation. Erbrich (2005) reported sensitivity field values for carbonate soils as high as $s=20$ and indicated that 'the laboratory samples have been substantially disturbed at some stage, leading to re-consolidation at lower void ratio'. Also, there are uncertainties on the rate of degradation of such sensitivity when under natural conditions.

Figure 8 reveals that $O_{c}=1.5$ offered the best overall fit when trying to replicate the bearing capacity profile of 'Exp Ref', with all the simulations starting from $O C R\left(K_{0}\right)$ conditions. The value was also verified by comparing the model-predicted undrained shear strength 
(Equation 9) with the distribution of undrained shear strength with depth as estimated using a miniature piezoball penetrometer. For the Laminaria soil parameters (Table 1) and the intact value of sensitivity $s_{i n i}=2.9$ (Figure 7), the back-analysed values of $O_{c}=1.5$ and $k=0.05$ led to an increase of $s_{u}$ with depth of $2.1 \mathrm{kPa} / \mathrm{m}$, which was close to $2.2 \mathrm{kPa} / \mathrm{m}$ measured by piezoball penetrometer. $K=0.05$ also offered the best fitting for the re-penetration curves post-consolidation, as detailed in the following parametric study. $s_{f}=1$ reflects the absence of any residual sensitivity after large remoulding and $A$ was shown to be in the range of 0.1 to 0.5 for most clays (Mašin 2007), however, it has only a minor influence, and a value of 0.2 was chosen here.

Even though both laboratory and centrifuge samples are considered to be in $K_{0}$ conditions, the two consolidation procedures occurring in the centrifuge and the triaxial tests are in fact different from each other. In particular, the centrifuge samples and the triaxial samples may potentially present different initial sensitivities, as the sample preparation processes involved are very different. Moreover, the formation of shear bands and strain localisation at the peak in the stress-strain path of triaxial tests was observed (Figure 6a), which make such tests unreliable for the calibration of parameters related to post-peak softening, particularly for large scale boundary value problems incorporating very large deformations as modelled here. Although already treated within hypoplasticity (Maier 2004), the modelling of such localisations was nonetheless outside the scope of this paper.

The set of parameters adopted in the simulations is given in Table 1.

\subsection{Validation}

The validation of the numerical model involved the retrospective simulations of centrifuge model data on spudcan installation with a pause during penetration from Bienen et al. (2015). 
Each centrifuge test involved (i) a stage of penetration to the target depth $w / D=1.0$ at a constant rate of $0.1 \mathrm{~mm} / \mathrm{s}$, with the normalised velocity $V=v D / c_{v}>100$ to guarantee undrained conditions, as discussed by Finnie (1993), Chung et al. (2006) and Cassidy (2012), (ii) a period of consolidation holding the load achieved at $w / D=1.0$ for a normalised time $T=c_{v} t / D^{2}$, and (iii) re-penetration at the same rate of $0.1 \mathrm{~mm} / \mathrm{s}$ to the target depth $w / D=2.0$. The permeability governing the rate of excess pore pressure build-up/dissipation was assumed to be isotropic and estimated as

$$
k_{w}=\gamma_{w} m_{v} c_{v}=\frac{\gamma_{w} c_{v} \lambda}{\sigma_{v}^{\prime}}
$$

where $\gamma_{w}$ is the unit weight of water, $m_{v}$ is the coefficient of volume compressibility of soil, $c_{v}$ the coefficient of vertical consolidation and $\sigma_{v}{ }^{\prime}$ the vertical effective stress. The coefficient of consolidation $c_{v}$ was fitted from the experimental oedometer test data as shown in Figure 9 with the following empirical equation

$$
c_{v}=\frac{\sigma_{v}^{\prime}}{10+0.478 \sigma_{v}^{\prime}}
$$

The units of $c_{v}$ and $\sigma_{v}{ }^{\prime}$ are $\mathrm{m}^{2} /$ year and $\mathrm{kPa}$, respectively. The numerical simulations generated using this relation show generally good comparability with the experimental interpretations in Figure 9.

Each test was labelled 'Exp $T=\ldots$ ' A further reference test with continuous penetration to $w / D=2.0$ was also performed and labelled 'Exp Ref'. The same procedure was adopted in the numerical analyses. The reference case is labelled 'Num Ref', the other analyses are labelled 'Num $T=\ldots$ '. All numerical analyses and centrifuge tests are summarised in Table 2. 
In order to widen the investigation and offer a better comparison with numerical results, a second series of Particle Image Velocimetry (PIV) tests was conducted in the UWA drum centrifuge (see Stanier \& White 2013 for details on the apparatus and modelling techniques adopted). A half spudcan model sliding against a Perspex window was used, with geometry similar to the above mentioned footing but $D=50 \mathrm{~mm}$ and no pore pressure transducers available (Bienen et al. 2015). This technique enabled the different failure mechanisms to be observed, expanding the set of information made available with the first series of tests, which only provided the histories of penetration resistance and excess pore pressures.

Figure 10 reports the bearing capacity $q=V_{s} / A_{s}$ profiles, where $V_{s}$ is the vertical load acting on the spudcan and $A_{s}$ the area at its widest section, for both experimental and numerical cases. In particular, a reference test with continuous penetration and three other tests involving a period of consolidation at depth $w / D=1.0$ with an increasing length $T=0.005$; 0.052; 0.746 are shown. Although the numerical cases start from an initial embedment of $0.5 D$, the reference case quickly realigns with its experimental counterpart, hence without affecting the consolidation stage at $w / D=1$ and showing a rapid transition to a fully localised mechanism (Hossain et al. 2005). In addition, the experimental and numerical reference cases provide similar resistance profiles, since the undrained shear strength profile deduced from the hypoplastic model, $2.1 \mathrm{kPa} / \mathrm{m}$, is close to the $2.2 \mathrm{kPa} / \mathrm{m}$ measured using the T-bar. The settlements observed during the consolidation stage at depth $w / D=1.0$, are significantly larger than those predicted numerically. The causes of such a difference are not fully understood. However, the normalised penetration rate immediately before consolidation in the experimental case is $V=120$ and well beyond the threshold of $V=80$ necessary to guarantee undrained conditions. The increased penetration rate at the onset of the load-hold period in the experiments can result in rate effects (Barbosa-Cruz 2007) leading to the onset of drainage being delayed whilst the normalised penetration rate falls from $\sim 120$ to $\sim 80$, 
before which excess pore-pressures are precluded from dissipating. For this reason, the larger experimental settlement may be seen as a combination of rate effects $(V>80)$ and consolidation $(V<80)$ components (Bienen et al. 2010), whereas the numerical analysis only accounts for the latter, as the model adopted is rate independent. For example, on average, in the experiments, $18 \%$ of the total load-hold induced settlement had already occurred before $\mathrm{V}<$ 80. Upon re-penetration after consolidation, a peak in the experimental curves is observed, followed by a continuous increase of the bearing capacity as the penetration advances towards $w / D=2.0$, behaviour already described by Bienen et al. (2015). The numerical analyses were able to reproduce the same trend, illustrating once again low risk for a potential consolidation-generated punch-through, as no bearing capacity reduction post-peak is observed, in contrast for example with the kaolin clay responses studied in Bienen \& Cassidy (2013). Similar numerical results (dotted lines of Figure 10) were achieved when adopting the centrifuge Boundary Conditions (BC) in terms of sample depth (from $20 D$ to $3.5 D$ ) and drainage conditions (halved drainage path), confirming the validity of centrifuge modelling in simulating real field conditions.

Figure 11 shows the dissipation during the consolidation stage of the excess pore pressure $\Delta u$ normalised by the bearing pressure $q, B_{q}=\Delta u / q$, plotted against the normalised consolidation time $T$, where $c_{v}$ is taken as the value defined by Equation (11) at the depth of the load reference point of the foundation at the onset of the load-hold period. The numerical dissipation curve 'Num $T=0.746$ ' and the three experimental dissipation curves present similar shape and they all start to dissipate at $T \sim 0.001$, with consolidation almost completed $\left(B_{q} \sim 0\right)$ when $T=0.746$ is reached. The longer the period of consolidation, the more excess pore pressures dissipated, which means a reduction in the void ratio around the spudcan and a consequent increase in shear strength. 
Numerical reproduction of centrifuge PIV tests carried out with a slightly different spudcan shape of diameter $D=50 \mathrm{~mm}$ at model scale enabled better visualisation. Figure 12 compares the numerical profiles of maximum undrained shear strength for current sensitivity $s$, calculated using Equation (9), immediately before (LHS) and after a consolidation period of $T=0.05$ (RHS). On the LHS a linear increase of undrained strength with depth at far field is observed, while the soil above the spudcan is highly remoulded. Instead, on the RHS, the excess pore pressure dissipation and consequent reduction in void ratio implies a significant increase in $s_{u}$ below the footing for approximately $1 \mathrm{D}$, as well as laterally.

The change in $s_{u}$ will affect the failure mechanism, as Figure 13 shows by comparing the normalised resultant velocity contours for experimental PIV data (LHS) and numerical simulations (RHS). Immediately before consolidation at $w / D=1$ (Figure 13a), both numerical and PIV results show a mechanism of comparable size, with soil flowing around the spudcan (Hossain et al. 2005). However, when restarting penetration after a pause of $T=0.05$ (Figure 13b), more soil under the spudcan is mobilised. Although there is a discrepancy in the volume of mobilised soil, the numerical analysis captured the soil above and below the spudcan moving downwards at a rate similar to the spudcan. Even upon further re-penetration of $0.5 \mathrm{D}$ (Figure 13c) and $0.75 \mathrm{D}$ (Figure 13d) the mechanism is still far from realigning with the localised flow-around in Figure 13a. Although it is gradually reducing in size, the soil volume with higher undrained strength trapped below the spudcan is obvious. This slow realignment with the reference case also contributes to explain the gaps observed in Figure 10 between the reference case and the post-consolidation profiles. 


\section{PARAMETRIC STUDY}

\subsection{Influence of $w / D, T, q$}

Unless consolidation is actively sought and hence planned for, neither (i) the depth at which the consolidation stage is going to take place (ii) duration of consolidation nor (iii) magnitude of pre-load which is held during the consolidation stage are known a priori. In order to provide a relevant database and facilitate the understanding of the effects of a pause during installation, a parametric study was performed. This included investigation of

- $\quad$ the effects of different consolidation depth $(w / D=1.0,1.5$ and 2.0);

- $\quad$ normalised consolidation time $(T=0.001,0.005,0.01,0.05,0.1$ and 0.5$)$;

- load ratio ( $q_{\text {cons }} / q_{\text {ref }}=0.5,0.75$ and 1.0), where $q_{\text {cons }}$ is the pressure held during the consolidation stage and $q_{\text {ref }}$ refers to the pressure applied when the spudcan is penetrated continuously to a particular depth.

This parametric study was designed to expand the existing experimental database in a systematic manner, covering a wider range of different types of consolidation stage, whilst still remaining representative of the qualitative trends already identified in the validation section.

The soil properties and spudcan dimensions are the same as in the validation section. All results are presented in terms of normalised time $T$, so that the results are indicative for different soils presenting different coefficients of consolidation - provided their characteristic behaviours are similar. In particular, the wide range of $T$ and $q_{\text {cons }} / q_{\text {ref }}$ investigated, aimed to cover possible field scenarios.

This parametric study allowed quantification of the expected trends on increasing spudcan capacity ( $q_{\text {peak }} / q_{\text {ref }}$, where $q_{\text {peak }}$ represents the bearing capacity recorded approximatively at 
the point where the re-penetration profiles change their curvature) with increasing consolidation time $T$, and larger gains for higher load ratios during the consolidation period. The enhancements of peak pressure at different consolidation depths are plotted in Figure 14 (black dots for $q_{\text {cons }} / q_{\text {ref }}=1.0$ ) against the normalised consolidation time $T$. The ratio is increased nearly linearly to the logarithm of $T$. Moreover, comparison of Figure 14a, b and c shows that the consolidation depth $w / D$ does not have an influence on the magnitude of such peak, given the same $T$ and $q_{\text {cons }} / q_{\text {ref }}$, with $q_{\text {peak }} / q_{\text {ref }}$ values always ranging from $1 \%$ to nearly $50 \%$. This confirms the independence of the ratio $q_{\text {peak }} / q_{\text {ref }}$ from the consolidation depth, within a realistic range of $w / D$, already shown by Bienen \& Cassidy (2013) for kaolin clay and Bienen et al. (2015) for carbonate silty clay, and also validates the experimental observations in kaolin clay of similar failure mechanisms for different consolidation depths of Stanier et al. (2014).

\subsection{Influence of $s, k$}

The strength of the numerical model featuring the hypoplastic soil constitutive model lies in the fact that soil behaviour with higher sensitivity, such as that expected from intact soil in the field, can now be investigated, and this is done here.

In order to cover the range of sensitivity expected in the field from carbonate deposits offshore Australia, the solid lines in Figure 15 present the bearing capacity profiles of four cases with increasing sensitivity $s_{i n i}=2.9 ; 5.8 ; 8.7 ; 20$ respectively, supported by observations of Erbrich (2005) of $s$ values as high as 20. The simulations involved a consolidation stage of $T=0.052$ commencing at $w / D=1.0$ and used the same $k=0.05$ and $O C R=1.205$. According to Figure 3 and 4, a reduced capacity would be expected in penetration from the tests with higher sensitivity at same $O C R$, as the final critical state is reached at a lower $q / p_{e}$ level. However, this behaviour is not observed here for two reasons: 
first, the very low rate of sensitivity degradation $(k=0.05)$ contributes to mitigate the effect of softening, i.e. softening does not have the chance to fully develop; second, higher values of $s$ imply soils existing at higher void ratio for a given stress (this follows from Equation 7), leading to partially drained conditions in penetration. This second reason is also supported by observation during the consolidation stage, by looking at the excess pore pressure dissipation curves of Figure 11. Soils with higher $s$ dissipate more quickly than the benchmark case with $s_{i n i}=2.9$, as the higher void ratio results in a higher permeability. In turn, this will have an effect on the re-penetration curves, with the $s_{i n i}=20$ case presenting the highest excess pore pressure dissipation and as a consequence the largest bearing capacity profile, followed by $s_{\text {ini }}=8.7, s_{\text {ini }}=5.8$ and $s_{i n i}=2.9$.

Sensitivity is not the only factor; the rate of degradation with accumulated shear strain plays an important role. While a value of $k=0.05$ fits the reconstituted carbonate silty clay used in the laboratory tests, values as high as 0.35 and 0.4 have been cited for Bothkennar and Pisa clays, respectively (Mašin 2007). The dashed lines of Figure 15 illustrate the effect a degradation rate of $k=0.2$ has on the behaviour. In this case the effect of softening becomes predominant over the enhanced bearing capacity due to consolidation. As a consequence, the case with lowest sensitivity presents the highest bearing capacity at $w / D=1.0$. Importantly, the shape of the penetration profile is significantly affected by the value of $s$. The spudcan load-penetration curves now show a 'nose', followed by a substantial reduction in resistance post peak. In these cases, consolidation has created punch-through risk that was not evident in the original soil profile, with the possibility of the spudcan penetrating rapidly at least another $0.8 D$ until bearing capacity similar to the peak value is met again. While the case of $s_{i n i}=2.9$ is not characteristic of punch-through, rapid leg run would be expected due to the almost constant resistance with depth. 
Figure 16 helps to further demonstrate the influence of strength softening rate, comparing sensitivity $s$ contour plots immediately before the consolidation stage at $w / D=1.0$ for $k=0.05$ (LHS) and $k=0.2$ (RHS), with both cases starting from an initial intact value of $s_{\text {ini }}=2.9$. While no soil reached the fully remoulded value with $k=0.05$ on LHS, a large portion of soil above and immediately below the spudcan reaches fully remoulded state $s=1$ on the RHS, and with a larger volume of soil that undergoes a more severe degradation process, according to Equation (5). The gap between the bearing capacity profiles generated using $k=0.05$ and $k=0.2$ is a result of the significant difference in distribution and concentration and softening generated during the penetration of the foundation.

Finally, Figure 17 and Figure 18 show the effects of different $q_{\text {cons }} / q_{\text {ref }}=0.5 ; 0.75 ; 1.0$ and $T=0.005 ; 0.052 ; 0.746$ on a soil in potentially natural conditions, with $s_{\text {ini }}=20$ and $k=0.05 ; 0.2$. In particular, Figure 17 shows that for $k=0.05$, a given period of consolidation ( $T=0.052$ in this case) will always be beneficial regardless of the fraction of load held in consolidation, with $q_{\text {peak }}$ proportional to the ratio $q_{\text {cons }} / q_{r e f}$ and $q$ increasing constantly in the following penetration post-peak. On the other hand, a higher degradation rate of $k=0.2$ will set the conditions for a potential punch-through, although with its severity mitigated by lower $q_{\text {cons }} / q_{\text {ref }}$ ratios, as both $q_{\text {peak }}$ and the extent of the uncontrolled leg penetration are reduced. The observed decrease in punch-through distance in particular, of roughly $50 \%$ from $q_{\text {cons }} / q_{\text {ref }}=1.0$ to $q_{\text {cons }} / q_{\text {ref }}=0.5$, is in line with the experimental observations of Bienen \& Cassidy (2013) for kaolin clay, soil that presents the same degradation rate $k=0.2$ (Ragni et al 2015). The three cases also lay on a common path after a given penetration, differing from the $k=0.2$ profiles where the influence of a different $q_{\text {cons }} / q_{\text {ref }}$ ratio is still present at $w / D=2.0$.

Figure 18 shows the effects of $T$ on the re-penetration profiles, where intuitively both $k=0.05$ (Figure 18a) and $k=0.2$ (Figure 18b) present $q_{\text {peak }}$ values proportional to $T$. An 
increasing gap between the re-penetration profiles and the reference case (dashed line) is observed when approaching $w / D=2.0$, confirming the long-lasting effect of enhanced bearing capacity post-consolidation described experimentally by Bienen et al. (2015) for the same carbonate silty clay. For $k=0.05$, an increment in $T$ leads to higher $q_{\text {peak }}$ values and more pronounced long-term beneficial effects, whereas for $k=0.2$ the severity of the punch-through is worsened by higher $T$, with the extent of uncontrolled leg penetration increasing from $0.25 D$ for $T=0.005$ to more than $0.7 D$ for $T=0.746$.

\subsection{Implications for practice}

The preceding figures illustrated the effects of a range of parameters influencing post-loadhold spudcan behaviour. In particular, the potential impact of high rates of the degradation of soil sensitivity emphasises the need to understand the soil conditions - in situ and undisturbed, as reconstituted samples may show differing behaviour. The parametric study presented in this paper indicates the range of behaviour that may be expected during spudcan penetration in soils where excess pore pressures (at least partially) dissipate during pauses in installation.

As a recommendation for practice it follows then, in addition to standard procedures to calibrate the parameters of the hypoplastic family of model, one needs to perform:

- Penetrometer (T-bar or ball) tests in situ during site investigation. This will provide an indication of the shear strength profile;

- Episodes of cyclic (T-bar or ball) penetrometer testing. This will provide an indication of the sensitivity;

- Penetrometer tests with pauses during penetration. This will provide information regarding the behaviour post-consolidation. 
The findings presented in this paper allow the identification of general trends in behaviour but cannot cover all possible parameter combinations. Specific predictions hence require analysis. Penetrometer data such as that recommended above allows calibration of:

- $O_{c}$ based on the initial penetration resistance;

- $s$ based on the cyclic penetrometer tests;

- $\quad k$ based on the behaviour during further penetration following a pause.

The implementation of the effects of sensitivity revealed the importance of modelling the correct soil characteristics: the results showed distinct behaviours when reconstituted rather than natural soil is modelled, and different sensitivity degradation rates led to different behaviours post-consolidation. This numerical study is a demonstration of a viable and cost effective approach to revealing aspects of natural soil behaviour for spudcan penetration-consolidation-penetration problems, when used in combination with reliable field data on natural soil and standard laboratory tests on reconstituted samples.

\section{CONCLUDING REMARKS}

The implementation of a hypoplastic constitutive model for structured clays in a large deformation finite element code was presented. The performance of the implementation has been validated through retrospective simulation of centrifuge test data, carried out on a carbonate silty clay recovered from the north-west shelf of Australia. The analyses reproduced the effects of pauses during spudcan installation, showing the increase in bearing capacity generated by such interruptions. A parametric study expanded the limited experimental database to cover a wide range of depth and length of load hold period, during which consolidation occurs due to dissipation of excess pore pressures. The implementation of sensitivity allowed soil in reconstituted and natural conditions to be modelled, revealing 
contrasting behaviours post-consolidation, that is dependent on the amount of sensitivity and its degradation rate. 


\section{REFERENCES}

Amodio, A., Chang, T.M., Kong, V. and Erbrich C.T. (2015). The effects of jack-up installation procedures on spudcan capacity in offshore carbonate sediments. Proc. $3^{\text {rd }}$ International Symposium on Frontiers in Offshore Geotechnics (ISFOG), Oslo, Norway, Vol. 2, pp. 1253-1260.

Atkinson, J.H. (2000). Non-linear soil stiffness in routine design. Géotechnique, Vol. 50, No. 5, pp. 487-508.

Barbosa-Cruz, E.R. (2007). Partial consolidation and breakthrough of shallow foundations in soft soil. PhD Thesis, University of Western Australia, Perth, Australia.

Baudet, B. and Stallebrass, S. (2004). A constitutive model for structured clays. Géotechnique, Vol. 54, No. 4, pp. 269-278.

Bienen, B., Gaudin, C. and Cassidy, M.J. (2010). Centrifuge study of the increase in undrained vertical bearing capacity of a circular shallow footing due to preloading. Proc. 7th International Conference on Physical Modelling in Geotechnics (ICPMG), Zurich, Switzerland, pp. 1019-1024.

Bienen, B. and Cassidy, M.J. (2013). Set up and resulting punch-through risk of jack-up spudcans during installation. Journal of Geotechnical and Geoenvironmental Engineering, Vol. 139, No. 12, pp. 2048-2059.

Bienen, B., Ragni, R., Cassidy, M.J. and Stanier, S.A. (2015). Effects of consolidation under a penetrating footing in carbonate silty clay. Journal of Geotechnical and Geoenvironmental Engineering, Vol. 141, No. 9, 04015040.

Brennan, R., Diana, H., Stonor, R.W.P., Hoyle, M.J.R., Cheng, C.P., Martin, D., Roper, R. (2006). Installing jackups in punch-through sensitive clays. Offshore Technology Conference (OTC), Houston, USA, OTC 18286.

Cassidy, M.J. (2012). Experimental observations of the penetration of spudcan footings in silt. Géotechnique, Vol. 62, No. 8, pp. 727-732.

Chung, S.F., Randolph, M.F. and Schneider, J.A. (2006). Effect of penetration rate on penetrometer resistance in clay. Journal of geotechnical and geoenvironmental engineering, Vol. 132, No. 9, pp. 1188-1196.

Cotecchia, F. and Chandler R.J. (2000). A general framework for the mechanical behaviour of clays. Géotechnique, Vol. 50, No. 4, pp. 431-447.

Dassault Systemes. (2012). Abaqus 6.12 User's manual.

Erbrich, C.T. (2005). Australian frontiers-spudcans on the edge. Proc. $1^{\text {st }}$ International Symposium on Frontiers in Offshore Geotechnics (ISFOG), Perth, Australia, Vol. 1, pp. 49-74.

Finnie, I.M.S. (1993). Performance of shallow foundations in calcareous soil. PhD Thesis. University of Western Australia, Perth, Australia. 
Gasparre, A. (2005). Advanced laboratory characterization of London clay. PhD Thesis, University of London, Imperial College of Science, Technology and Medicine, London, UK.

Gudehus, G. (1996). A comprehensive constitutive equation for granular materials. Soils and Foundations, Vol. 36, No. 1, pp. 1-12.

Gudehus, G., Amorosi, A., Gens, A., Herle, I., Kolymbas, D., Mašín, D., Muir Wood, D., Nova, R., Niemunis, A., Pastor, M., Tamagnini, C. and Viggiani, G. (2008). The soilmodels.info project. International Journal for Numerical and Analytical Methods in Geomechanics, Vol. 32, No. 12, pp. 1571-1572.

Hossain, M.S., Hu, Y., Randolph, M.F. and White, D.J. (2005). Limiting cavity depth for spudcan foundations penetrating clay. Géotechnique, Vol. 55, No. 9, pp. 679-690.

Hu, Y. and Randolph, M.F. (1998). A practical numerical approach for large deformation problems in soil. International Journal of Numerical and Analytical Methods in Geomechanics, Vol. 22, No. 5, pp. 327-350.

International Organization for Standardization (ISO) (2012) - Petroleum and natural gas industries - Site-specific assessment of mobile offshore unit - Part 1: Jack-ups. Geneva, Switzerland.

Maier, T. (2004). Comparison of non-local and polar modelling of softening in hypoplasticity. International Journal for Numerical and Analytical Methods in Geomechanics, Vol. 28, No. 3, pp. 251-268.

Mašín, D. (2005). A hypoplastic constitutive model for clays. International Journal for Numerical and Analytical Methods in Geomechanics, Vol. 29, No. 4, pp. 311-336.

Mašín, D. (2007). A hypoplastic constitutive model for clays with meta-stable structure. Canadian Geotechnical Journal, Vol. 44, No. 3, pp. 363-375.

Mašín, D. (2012). Hypoplastic Cam-clay model. Géotechnique, Vol. 62, No. 6, pp. 549-553.

Mašín, D. (2014). Clay hypoplasticity model including stiffness anisotropy. Géotechnique, Vol. 64, No. 3, pp. 232-238.

Ragni, R., Wang, D., Mašín, D., Cassidy, M.J. and Bienen, B. (2015b). Modelling the effects of pauses during spudcan penetration on the further installation behaviour. Proc. $15^{\text {th }}$ International Conference of Jack-up Platform, London, UK, Paper No. 15.

Randolph, M.F., Hefer, P.A., Geise, J.M. and Watson, P.G. (1998). Improved seabed strength profiling using T-bar penetrometer. Proc International Conference on Offshore Site Investigation and Foundation Behaviour - "New Frontiers", Society for Underwater Technology, London, UK, pp. 221-235.

Rott, J., Mašín, D., Boháč, J., Krupička, M. and Mohyla, T. (2015). Evaluation of $K_{0}$ in stiff clay by back-analysis of convergence measurements from unsupported cylindrical cavity. Acta Geotechnica, Vol. 10, No. 6, pp. 719-733. 
Stanier, S.A., and White, D.J. (2013). Improved image-based deformation measurement in the centrifuge environment. ASTM geotechnical testing journal, Vol. 36, No. 6, pp.915928.

Stanier, S.A., Ragni, R., Bienen, B. and Cassidy, M.J. (2014). Observing the effects of sustained loading on spudcan footings in clay. Géotechnique, Vol. 64, No. 11, pp. 918-926.

Wang, D., Hu, Y. and Randolph, M.F. (2008). Effect of loading rate on the uplift capacity of plate anchors. Proc. 18th Int. Offshore and Polar Eng. Conf., Vol. 2, pp. 727-731.

Wang, D. and Bienen, B. (2015). Numerical investigation of penetration of a large diameter footing into normally consolidated permeable clay with a consolidation phase. Géotechnique, currently under review.

Wang, D., Bienen, B., Nazem, M., Tian, Y., Zheng, J., Pucker, T., \& Randolph, M.F. (2015). Large deformation finite element analyses in geotechnical engineering. Computers and Geotechnics, Vol. 65, pp. 104-114.

Von Wolffersdorff P.A., (1996). A hypoplastic relation for granular materials with a predefined limit state surface. Mechanics of Cohesive-Frictional Materials, Vol. 1, pp. 251-271. 


\section{NOMENCLATURE}

a acceleration level

A volumetric/shear strain effect on sensitivity degradation

$A_{s} \quad$ area of the spudcan

$B_{q} \quad$ normalised excess pore pressures

$C_{v} \quad$ coefficient of vertical consolidation

D spudcan diameter

e void ratio

$f_{d} \quad$ scalar factor of the hypoplastic constitutive law

$f_{s} \quad$ scalar factor of the hypoplastic constitutive law

$k \quad$ sensitivity degradation rate

$K_{0} \quad$ earth pressure coefficient at rest

$k_{w} \quad$ soil permeability

$\boldsymbol{L}$ fourth-order constitutive tensor

M slope of the critical state line

$m_{v} \quad$ coefficient of volume compressibility

N second-order constitutive tensor

$N \quad$ intercept of the isotropic compression line at $p^{\prime}=1 \mathrm{kPa}$

$N_{\text {ball }} \quad$ piezoball penetrometer factor

$O_{c} \quad$ position of the critical state line

OCR over-consolidation ratio

$p^{\prime} \quad$ mean effective stress

$p_{c} \quad$ pressure at critical state

$p_{e} \quad$ Hvorslev equivalent pressure

$p_{r} \quad$ reference stress

$q \quad$ bearing capacity

$q_{\text {cons }}$ pressure applied in consolidation

$q_{\text {peak }}$ bearing capacity peak recorded in re-penetration

$q_{\text {ref }}$ pressure applied in continuous spudcan penetration 
$r \quad$ bulk modulus in isotropic compression at NCL over shear modulus

$S \quad$ sensitivity

$s_{f} \quad$ final sensitivity

$s_{i n i} \quad$ initial sensitivity

$s_{u} \quad$ undrained shear strength

$s_{u, i n i} \quad$ initial intact undrained shear strength

$t \quad$ dimensional time

$T \quad$ normalised consolidation time

$u \quad$ excess pore pressures

$\checkmark \quad$ penetration rate

$V \quad$ normalised penetration rate

$V_{s} \quad$ vertical load acting on the spudcan

w penetration depth

$\gamma^{\prime} \quad$ specific unit weight of the soil

$\gamma_{w} \quad$ unit weight of water

$\Delta u \quad$ excess pore pressure variation

$\dot{\varepsilon} \quad$ Euler stretching tensor

$\dot{\varepsilon}^{d} \quad$ damage strain rate

$\dot{\varepsilon}^{s} \quad$ shear strain rate

$\dot{\varepsilon}^{v} \quad$ volumetric strain rate

$\kappa^{*} \quad$ slope of the unloading-reloading line

$\lambda^{*} \quad$ slope of the normally compressed line

$v \quad$ control parameter on shear modulus

$\dot{\sigma} \quad$ objective stress rate

$\sigma_{v}{ }^{\prime} \quad$ vertical effective stress

$\varphi_{c} \quad$ critical state friction angle 


\section{LIST OF TABLES}

Table 1: Hypoplastic model parameters for Laminaria carbonate silty clay.

Table 2: Overview of numerical analyses and experimental tests.

\section{LIST OF FIGURES}

Figure 1: Schematic of a typical spudcan preload force and penetration depth time history during jack-up installation.

Figure 2: Numerical soil domain and mesh strategy around the spudcan.

Figure 3: Hypoplastic bi-logarithmic compression law for structured clays.

Figure 4: Influence of $O_{c}$ on a) the shape of the Asymptotic State Boundary Surface and on the undrained stress paths of $K_{0}$ consolidated carbonate silty clay; b) stress-strain curves for the same undrained shear tests, demonstrating also the effect of $O_{c}$ on undrained shear strength.

Figure 5: Results of oedometric test on carbonate silty clay from reconstituted sample and corresponding numerical simulation.

Figure 6: Results of triaxial tests on carbonate silty clay on a) stress-strain plane, b) mean effective-deviator stress path.

Figure 7: Episodes of cyclic T-bar penetration in centrifuge environment showing sensitivity degradation.

Figure 8: Effect of parameter $O_{c}$ on the spudcan bearing capacity.

Figure 9: Coefficient of vertical consolidation obtained from oedometer test. 
Figure 10: Vertical profiles of spudcan bearing capacity $q=V / A$ for centrifuge experimental data and numerical retrospective simulations.

Figure 11: Time history of normalised excess pore pressure dissipation $B_{q}=\Delta u / q$ against normalised time $T$ in semi-logarithmic scale.

Figure 12: Undrained soil shear strength contour plot for current sensitivity from numerical simulation immediately before (LHS) and immediately after (RHS) a period of consolidation $T=0.05$

Figure 13: Normalised resultant velocity contours for experimental PIV data (LHS) and numerical simulations (RHS) a) immediately before consolidation $T=0.05$, b) immediately after consolidation, c) $0.5 \mathrm{D}$ after consolidation, d) $0.75 \mathrm{D}$ after consolidation.

Figure 14: Increase in bearing capacity with normalised time $T$ for a) $w / D=1.0$; b) $w / D=1.5 ;$ c) $w / D=2.0$

Figure 15: Effects of sensitivity $s$ and degradation rate $k$ on the vertical bearing capacity profiles for numerical simulations.

Figure 16: Sensitivity degradation contour plot for $k=0.05$ (LHS) and $k=0.2$ (RHS), starting from intact sensitivity $s_{i n i}=2.9$.

Figure 17: Effects of different load sustained in consolidation $q_{\text {cons }} / q_{\text {ref }}$ on the vertical bearing capacity profiles of natural soil with $s_{i n i}=20$.

Figure 18: Effects of different consolidation length $T$ on the vertical bearing capacity profiles of natural soil with $s_{i n i}=20$. 


\begin{tabular}{|c|c|c|c|c|}
\hline \multicolumn{5}{|c|}{ Basic Model Parameters } \\
\hline$\varphi_{c}$ & $N$ & $\lambda^{*}$ & $\boldsymbol{\kappa}^{*}$ & $v$ \\
\hline $\begin{array}{l}\text { CS } \\
\text { friction } \\
\text { angle }\end{array}$ & $\begin{array}{l}\text { NCL for } \\
p^{\prime}=1 \mathrm{kPa}\end{array}$ & $\begin{array}{l}\text { NCL } \\
\text { slope }\end{array}$ & $\begin{array}{l}\text { Unloading-reloading } \\
\text { slope }\end{array}$ & $\begin{array}{l}\text { Control } \\
\text { on shear } \\
\text { modulus }\end{array}$ \\
\hline $34^{\circ}$ & 1.697 & 0.114 & 0.013 & 0.1 \\
\hline
\end{tabular}

\begin{tabular}{cccc}
\hline \multicolumn{4}{c}{ Sensitivity Parameters } \\
\hline $\begin{array}{c}\boldsymbol{k} \text { ini } \\
\text { Initial } \\
\text { sensitivity }\end{array}$ & $\begin{array}{c}\boldsymbol{k} \\
\text { sensitivity } \\
\text { degradation } \\
\text { rate }\end{array}$ & $\begin{array}{c}\text { Vol/shear } \\
\text { strains } \\
\text { effect }\end{array}$ & $\begin{array}{c}\boldsymbol{s}_{\boldsymbol{f}} \\
\text { Final } \\
\text { sensitivity }\end{array}$ \\
\hline 2.9 & 0.05 & 0.2 & 1 \\
\hline
\end{tabular}

\begin{tabular}{|c|c|c|}
\hline \multicolumn{3}{|c|}{ Consolidation Parameters } \\
\hline$K_{0}$ & $O_{c}$ & OCR \\
\hline Earth & CSL & Over \\
\hline pressure & position & Consolidation \\
\hline $\begin{array}{c}\text { coeff. at } \\
\text { rest }\end{array}$ & & Ratio \\
\hline 0.441 & 1.5 & 1.205 \\
\hline
\end{tabular}

Table 1: Hypoplastic model parameters for Laminaria carbonate silty clay. 


\begin{tabular}{|c|c|c|c|c|c|c|c|c|c|}
\hline \multicolumn{10}{|c|}{ Verification } \\
\hline \multicolumn{4}{|c|}{ Test } & \multicolumn{4}{|c|}{ Consolidation } & \multicolumn{2}{|c|}{$\begin{array}{l}\text { Sensitivity } \\
\text { Parameters }\end{array}$} \\
\hline Method & $\begin{array}{l}\mathbf{D}_{\text {model }} \\
(\mathrm{mm})\end{array}$ & Exp & Num & $\begin{array}{c}\text { Prototype } t \\
\text { (years) }\end{array}$ & $T=c_{v} t / D^{2}$ & w/D & $\mathbf{q}_{\text {cons }} / \mathbf{q}_{\text {ref }}$ & $s_{\text {ini }}$ & $k$ \\
\hline \multirow{4}{*}{$\begin{array}{c}\text { Full } \\
\text { spudcan }\end{array}$} & \multirow{4}{*}{60} & $\checkmark$ & $\checkmark$ & $-($ Ref $)$ & - (Ref) & \multirow{4}{*}{1.0} & \multirow{4}{*}{1.0} & \multirow{5}{*}{2.9} & \multirow{5}{*}{0.05} \\
\hline & & $\checkmark$ & $\checkmark$ & 0.46 & 0.005 & & & & \\
\hline & & $\checkmark$ & $\checkmark$ & 4.74 & 0.052 & & & & \\
\hline & & $\checkmark$ & $\checkmark$ & 67.99 & 0.746 & & & & \\
\hline PIV & 50 & $\checkmark$ & $\checkmark$ & 3.23 & 0.05 & 1.0 & 1.0 & & \\
\hline \multicolumn{10}{|c|}{ Parametric Study } \\
\hline \multicolumn{4}{|c|}{ Test } & \multicolumn{4}{|c|}{ Consolidation } & \multicolumn{2}{|c|}{$\begin{array}{c}\text { Sensitivity } \\
\text { Parameters }\end{array}$} \\
\hline Method & $\begin{array}{l}\mathbf{D}_{\text {model }} \\
(\mathrm{mm})\end{array}$ & Exp & Num & $\begin{array}{c}\text { Prototype } t \\
\text { (years) }\end{array}$ & $T=c_{v} t / D^{2}$ & w/D & $\mathbf{q}_{\text {cons }} / \mathbf{q}_{\text {ref }}$ & $s_{\text {ini }}$ & $k$ \\
\hline \multirow{21}{*}{$\begin{array}{c}\text { Full } \\
\text { spudcan }\end{array}$} & \multirow{21}{*}{60} & & $\checkmark$ & 0.09 & & 1.0 & & \multirow{12}{*}{2.9} & \multirow{12}{*}{0.05} \\
\hline & & & $\checkmark$ & 0.08 & 0.001 & 1.5 & 0.5, 0.75, & & \\
\hline & & & $\checkmark$ & 0.08 & & 2.0 & & & \\
\hline & & & $\checkmark$ & 0.46 & & 1.0 & & & \\
\hline & & & $\checkmark$ & 0.42 & 0.005 & 1.5 & 0.5, 0.75, & & \\
\hline & & & $\checkmark$ & 0.40 & & 2.0 & & & \\
\hline & & & $\checkmark$ & 0.91 & \multirow{3}{*}{0.01} & 1.0 & \multirow{3}{*}{$\begin{array}{c}0.5,0.75 \\
1.0\end{array}$} & & \\
\hline & & & $\checkmark$ & 0.84 & & 1.5 & & & \\
\hline & & & $\checkmark$ & 0.80 & & 2.0 & & & \\
\hline & & & $\checkmark$ & 4.55 & \multirow{3}{*}{0.05} & 1.0 & \multirow{3}{*}{$\begin{array}{c}0.5,0.75 \\
1.0\end{array}$} & & \\
\hline & & & $\checkmark$ & 4.19 & & 1.5 & & & \\
\hline & & & $\checkmark$ & 4.00 & & 2.0 & & & \\
\hline & & & $\checkmark$ & 0.46 & 0.005 & 1.0 & 1.0 & 20 & $\begin{array}{c}0.05, \\
0.2\end{array}$ \\
\hline & & & $\checkmark$ & 4.74 & 0.052 & 1.0 & $\begin{array}{c}0.5,0.75 \\
1.0\end{array}$ & $\begin{array}{c}2.9, \\
5.8, \\
8.7 \\
20\end{array}$ & $\begin{array}{c}0.05, \\
0.2\end{array}$ \\
\hline & & & $\checkmark$ & 67.99 & 0.746 & 1.0 & 1.0 & 20 & $\begin{array}{c}0.05, \\
0.2\end{array}$ \\
\hline & & & $\checkmark$ & 9.11 & \multirow{3}{*}{0.1} & 1.0 & \multirow{3}{*}{$\begin{array}{c}0.5,0.75 \\
1.0\end{array}$} & \multirow{6}{*}{2.9} & \multirow{6}{*}{0.05} \\
\hline & & & $\checkmark$ & 8.37 & & 1.5 & & & \\
\hline & & & $\checkmark$ & 8.00 & & 2.0 & & & \\
\hline & & & $\checkmark$ & 45.57 & \multirow{3}{*}{0.5} & 1.0 & \multirow{3}{*}{$\begin{array}{c}0.5,0.75 \\
1.0\end{array}$} & & \\
\hline & & & $\checkmark$ & 41.86 & & 1.5 & & & \\
\hline & & & $\checkmark$ & 40.00 & & 2.0 & & & \\
\hline
\end{tabular}

Table 2: Overview of numerical analyses and experimental tests. 


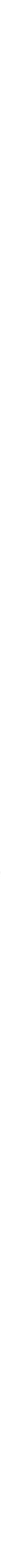

Path followed in experiments 


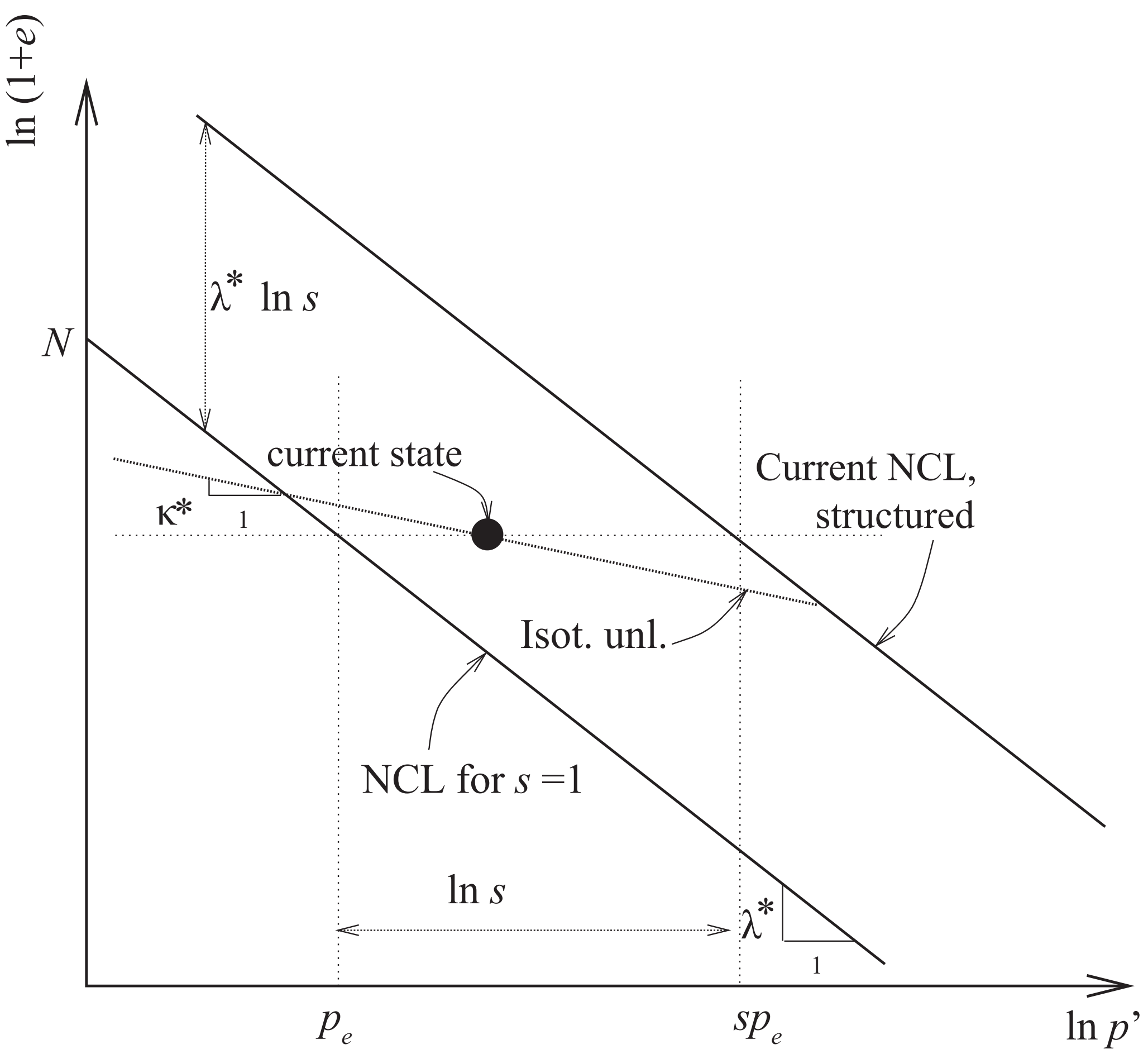




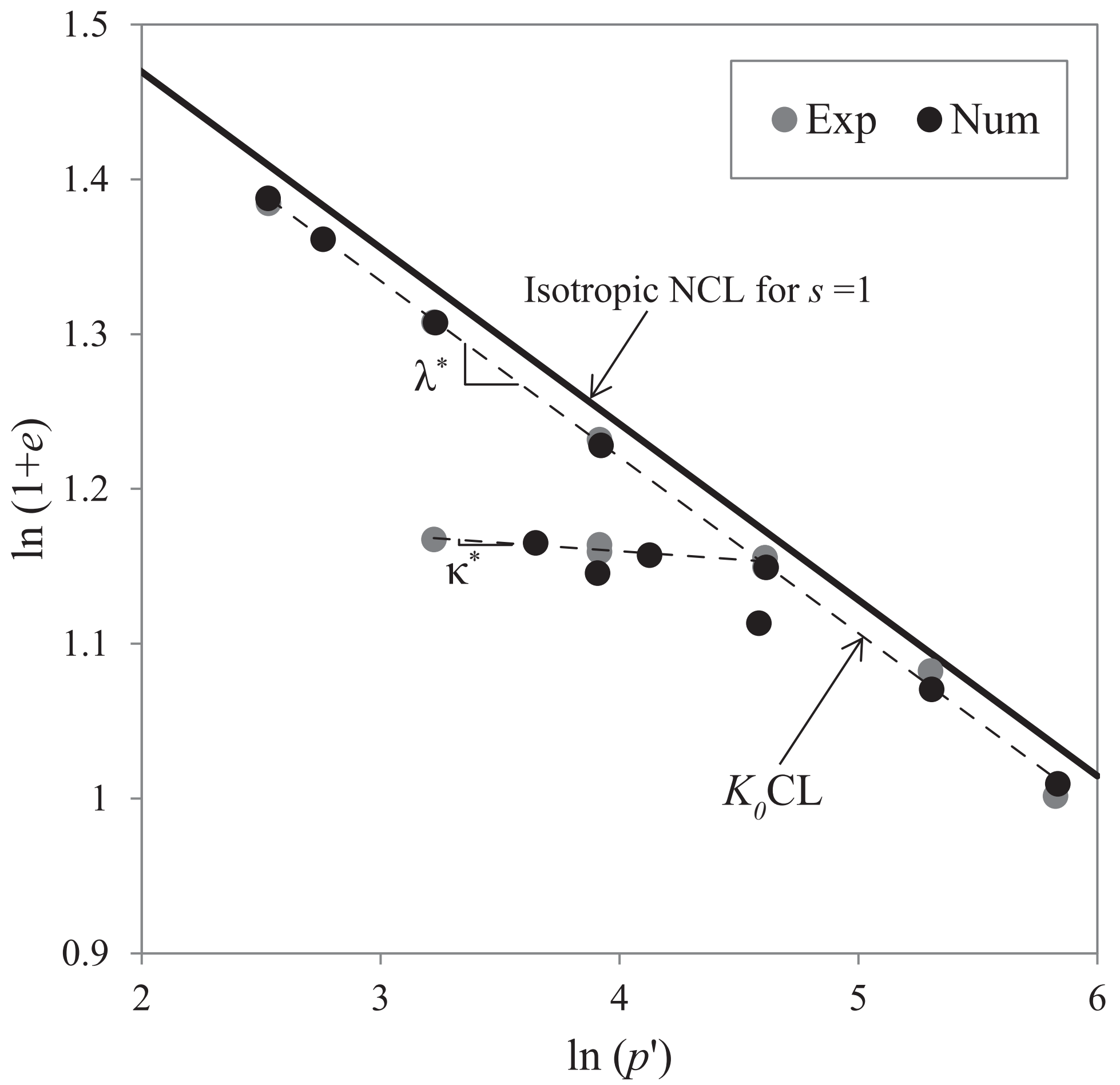




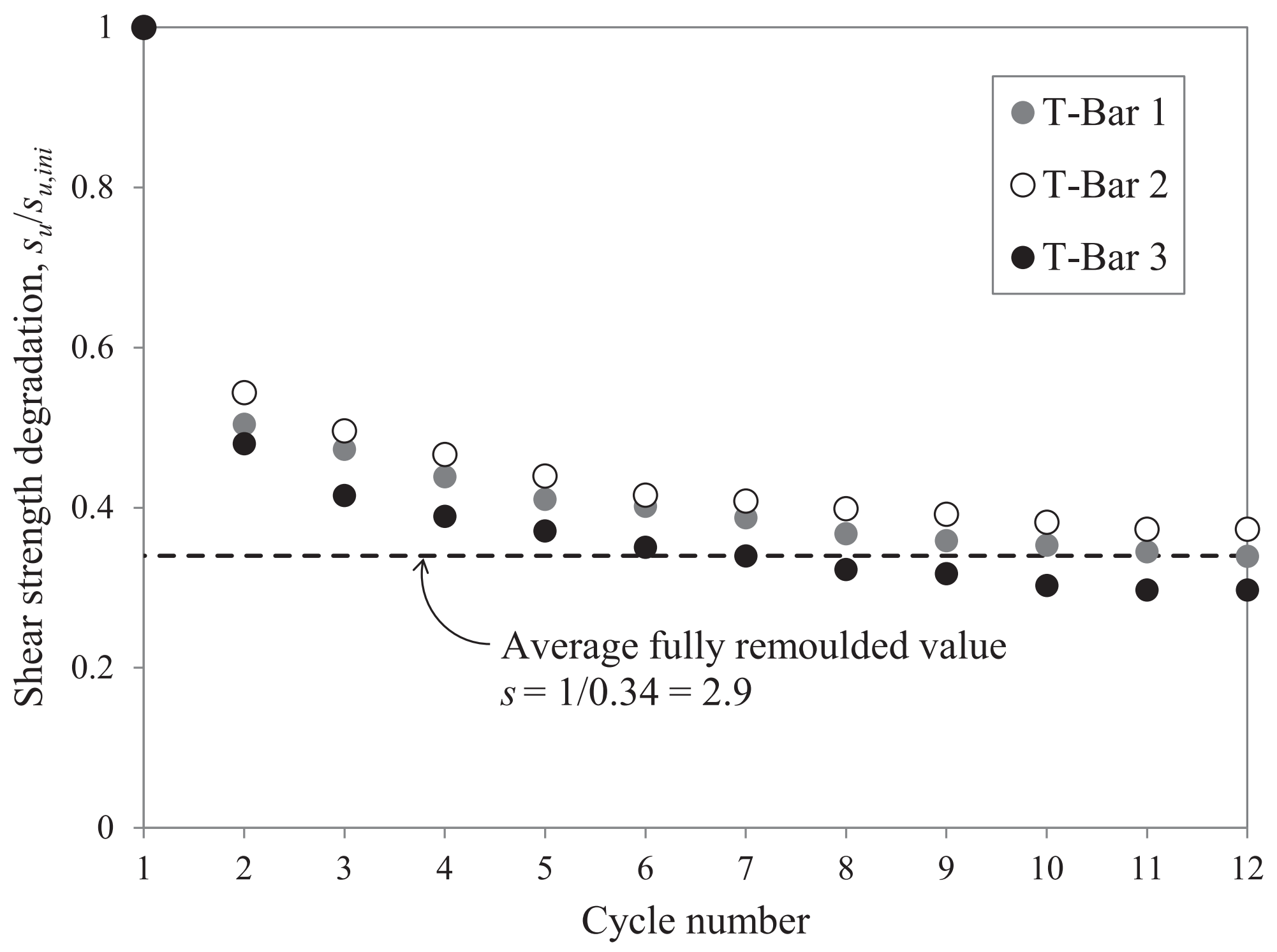


Bearing pressure, $q(\mathrm{kPa})$

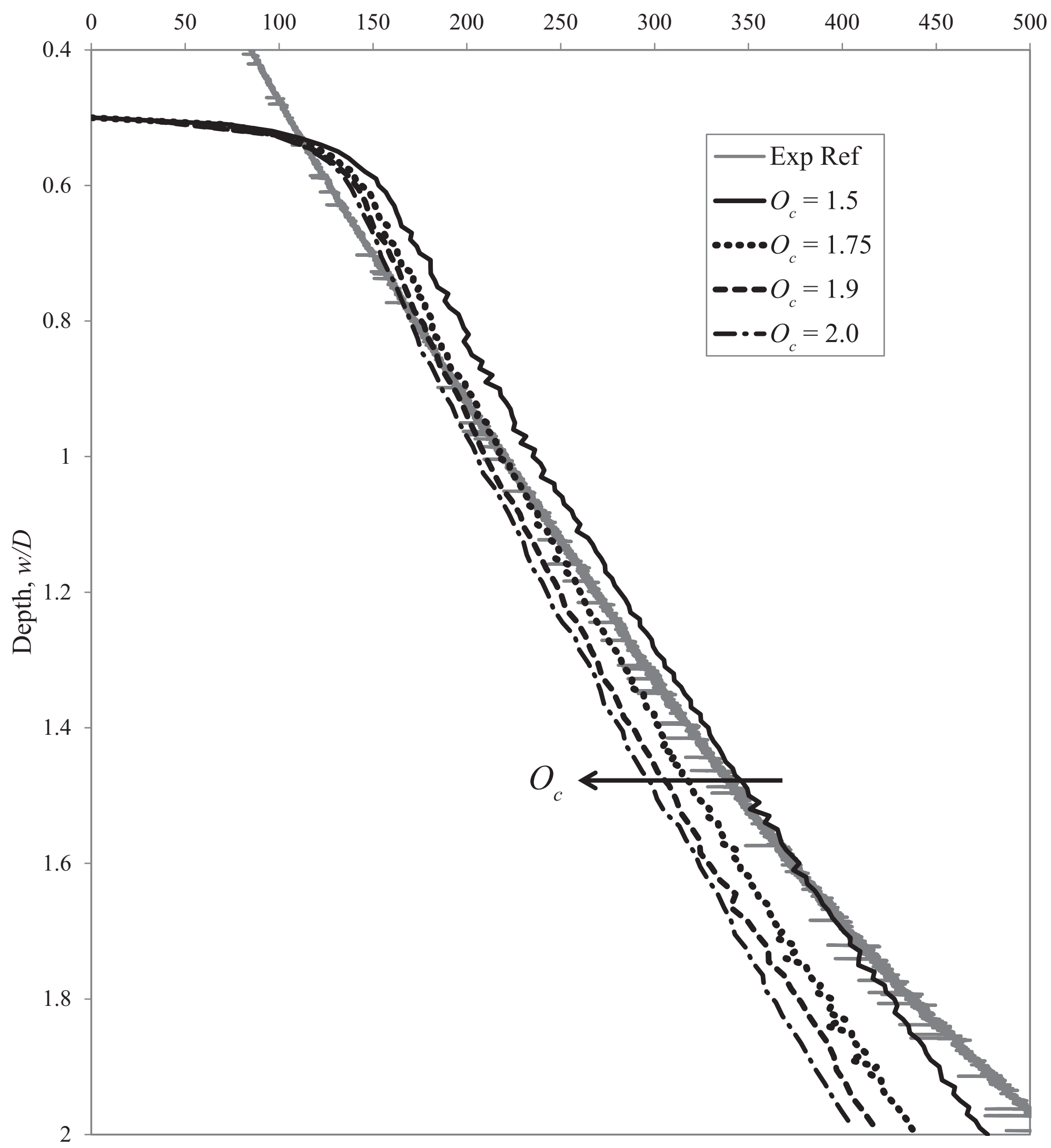




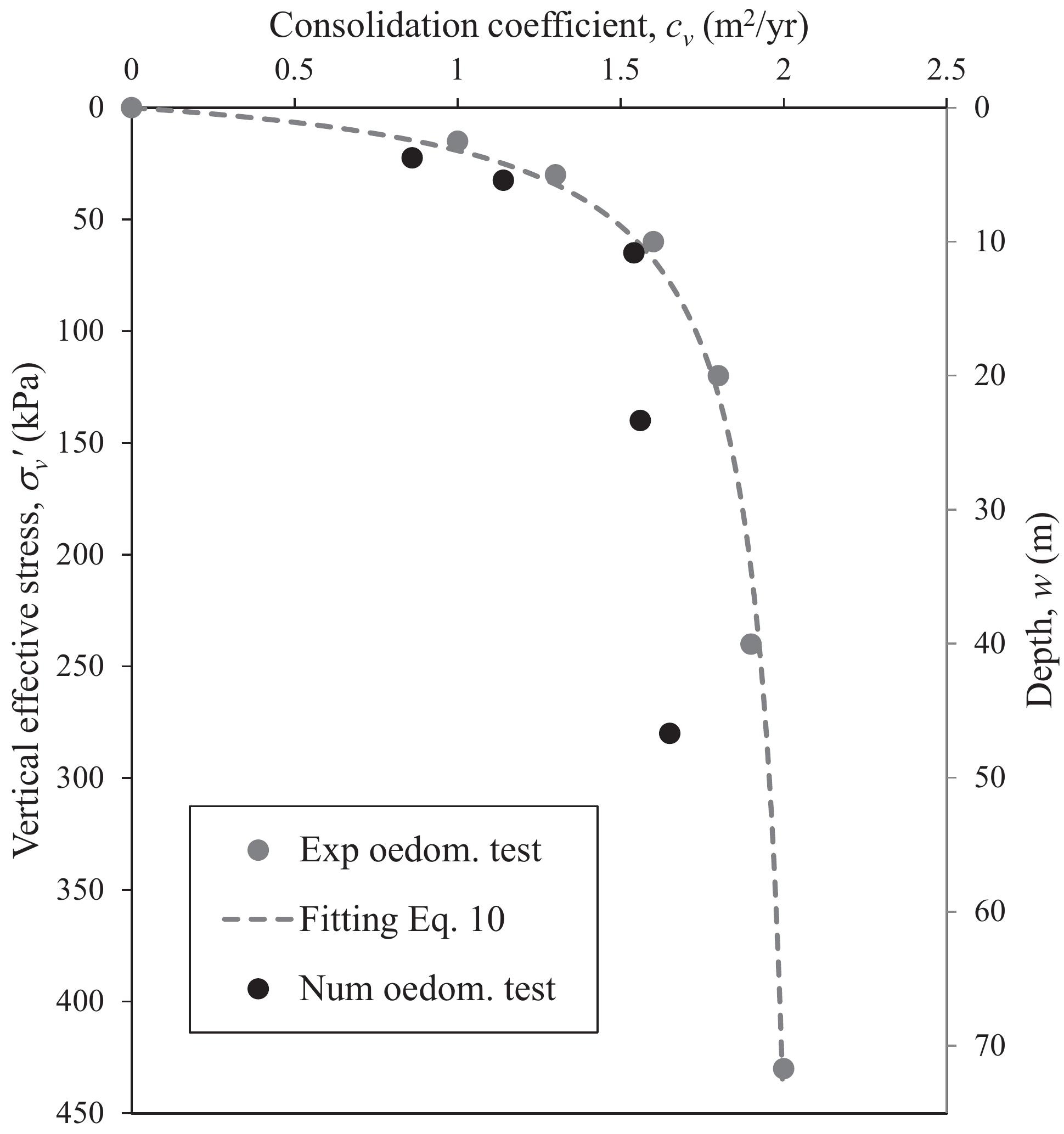




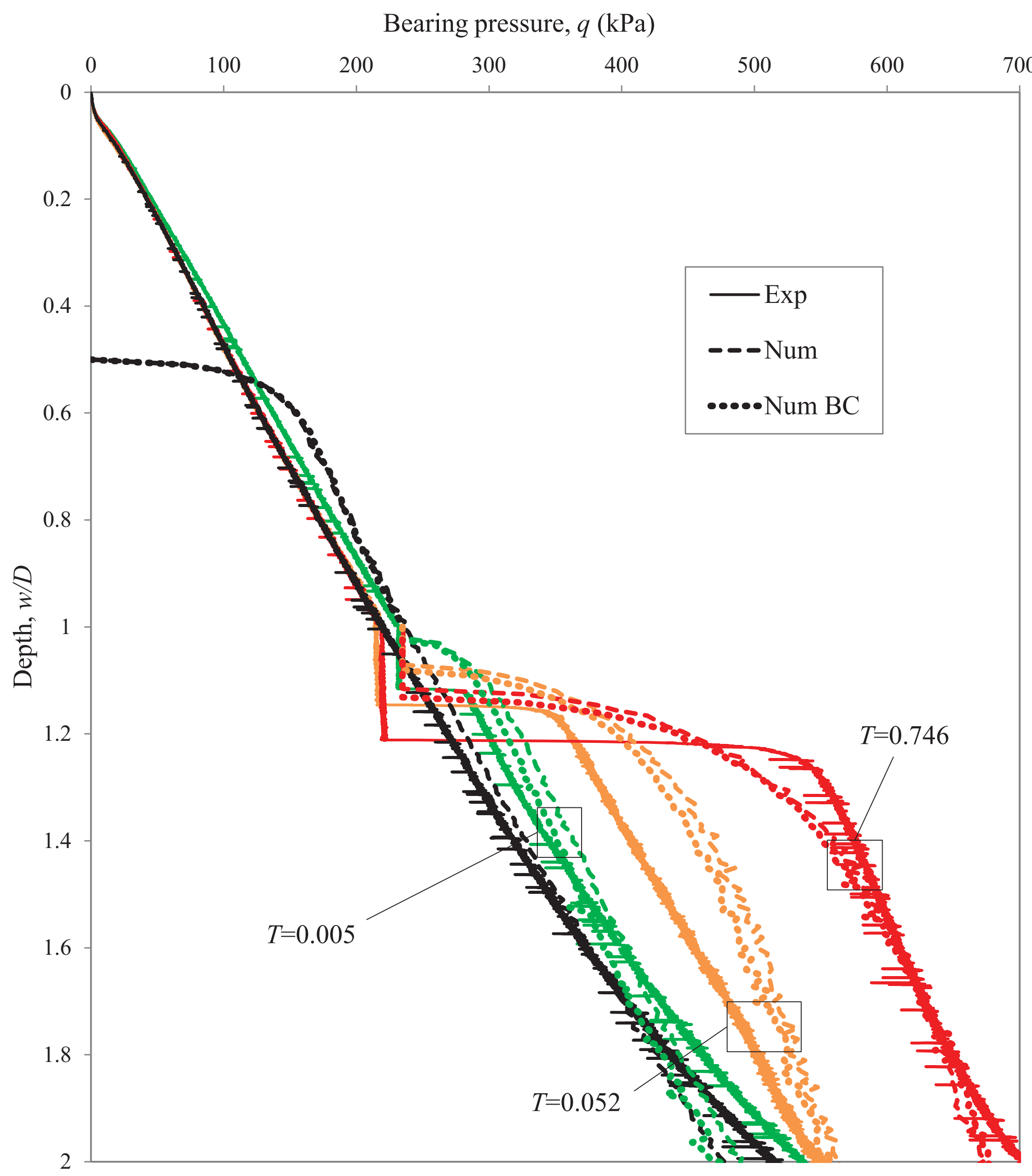




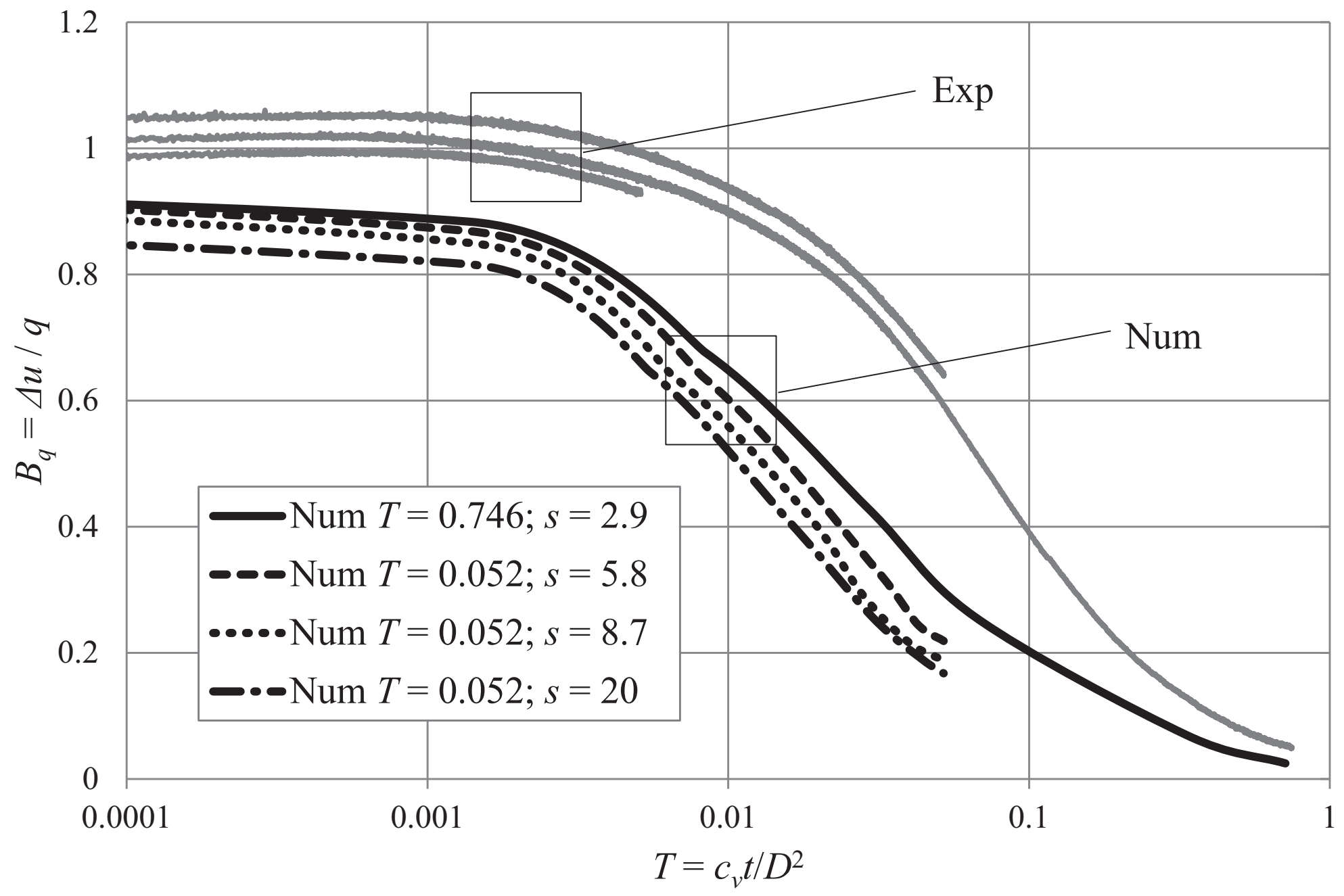




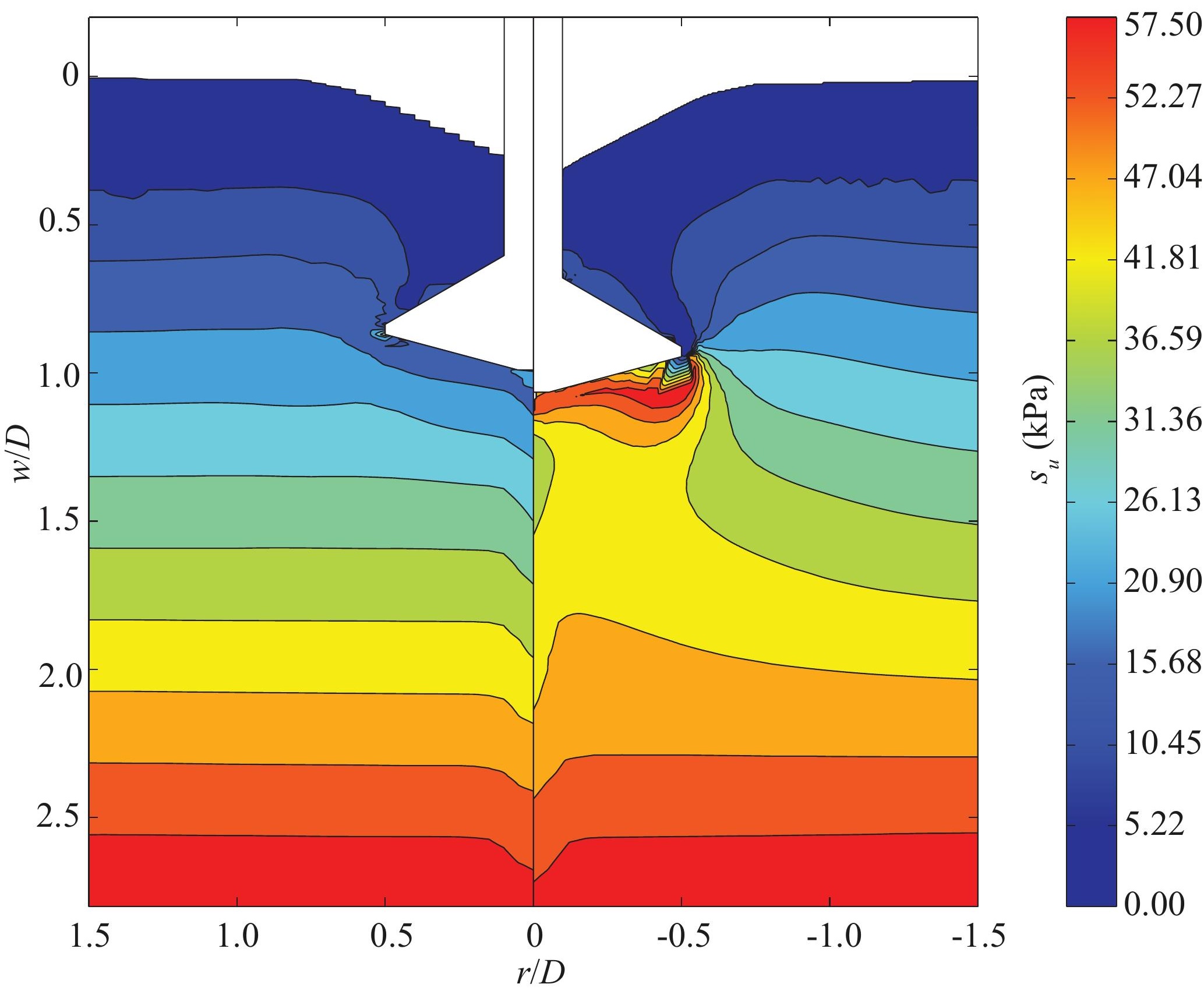


Figuredat
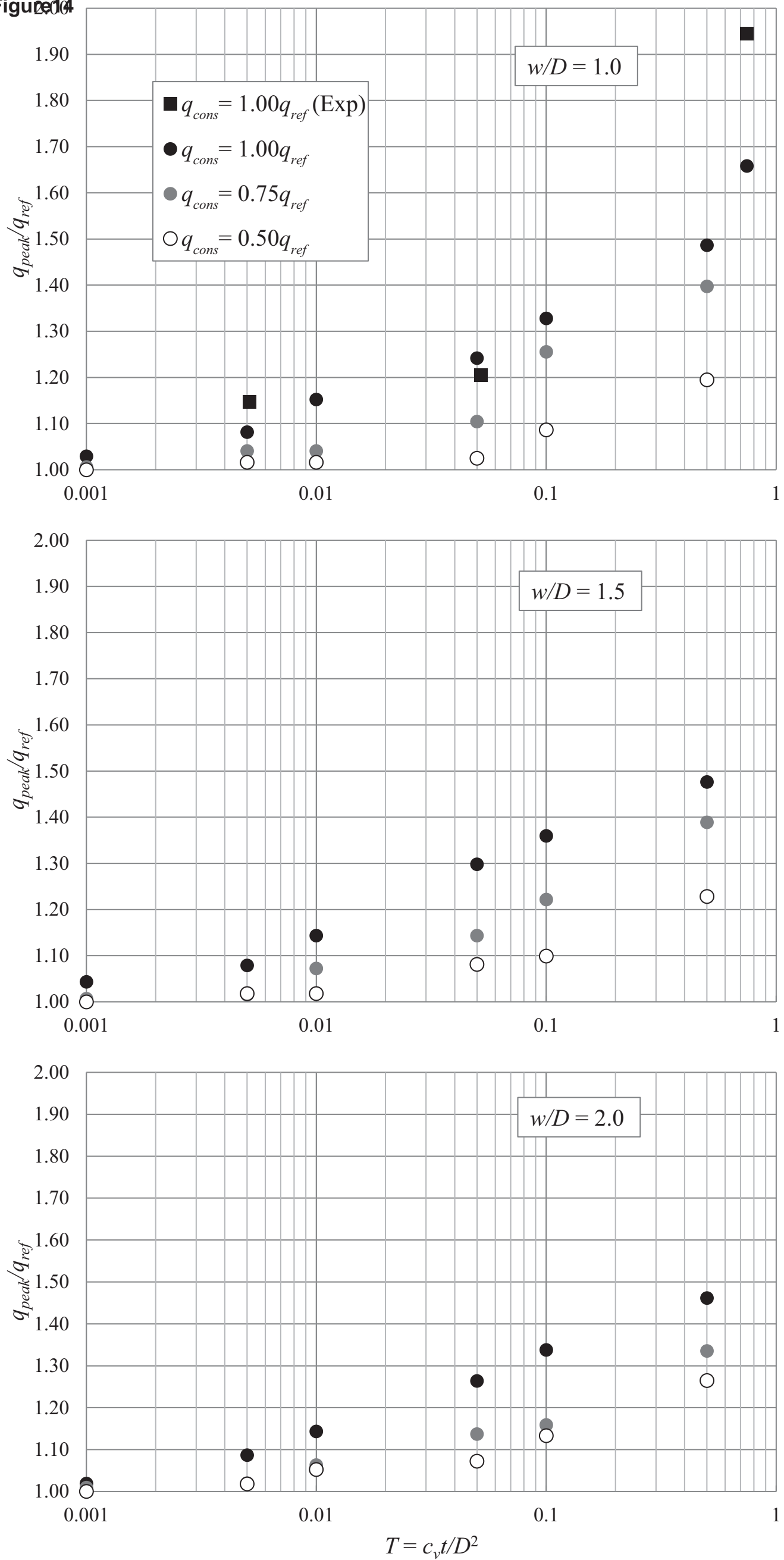


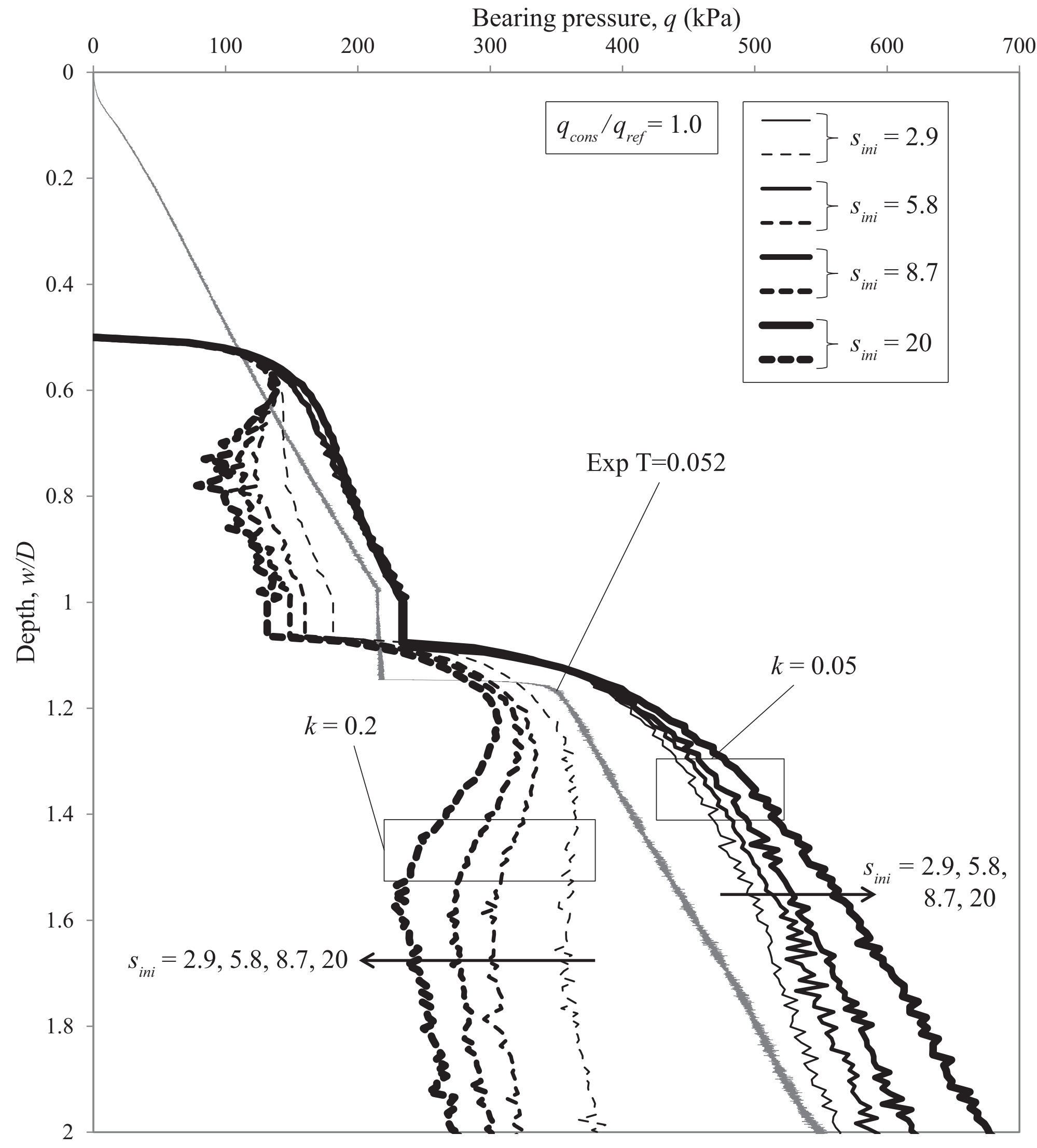




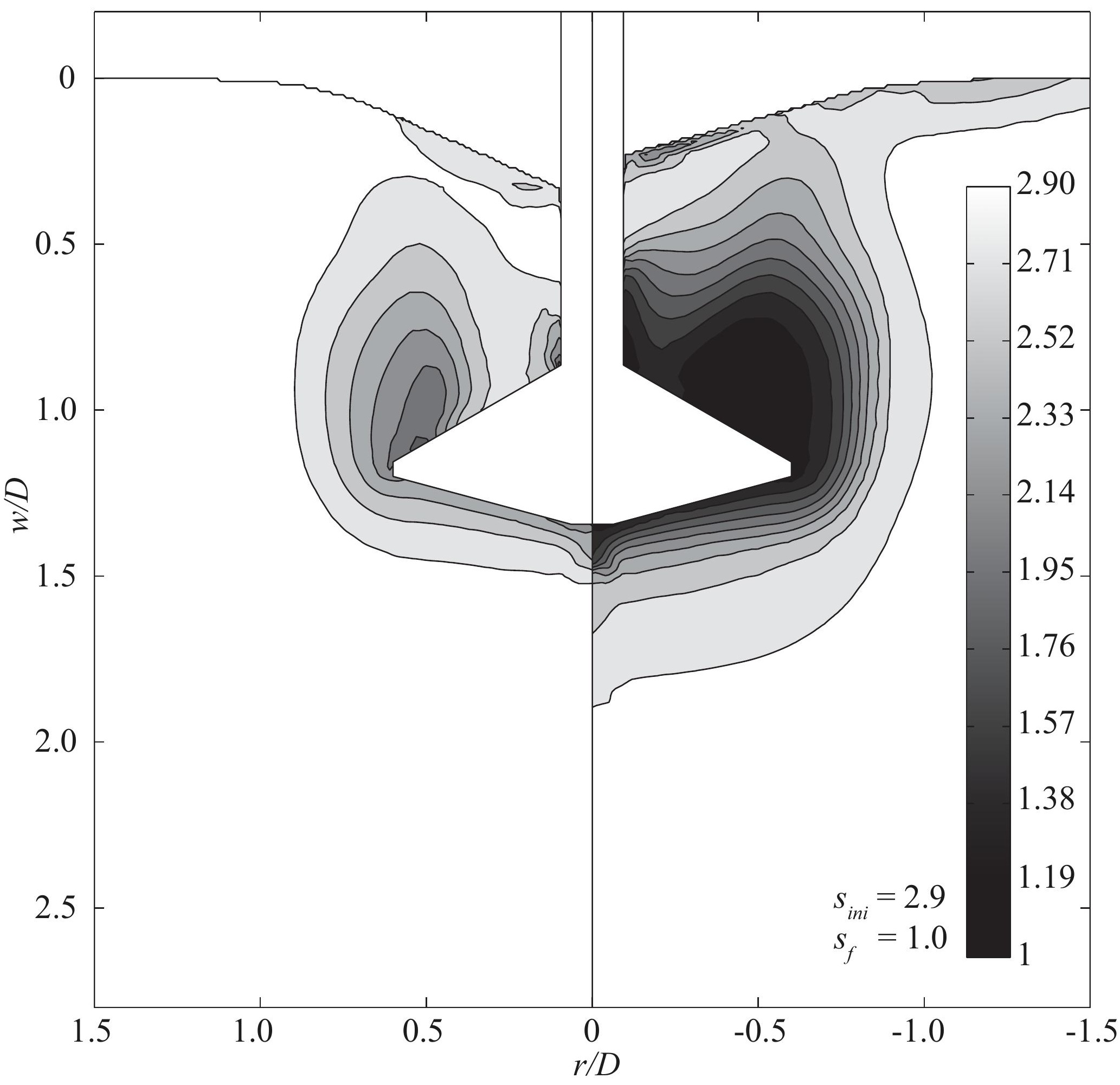




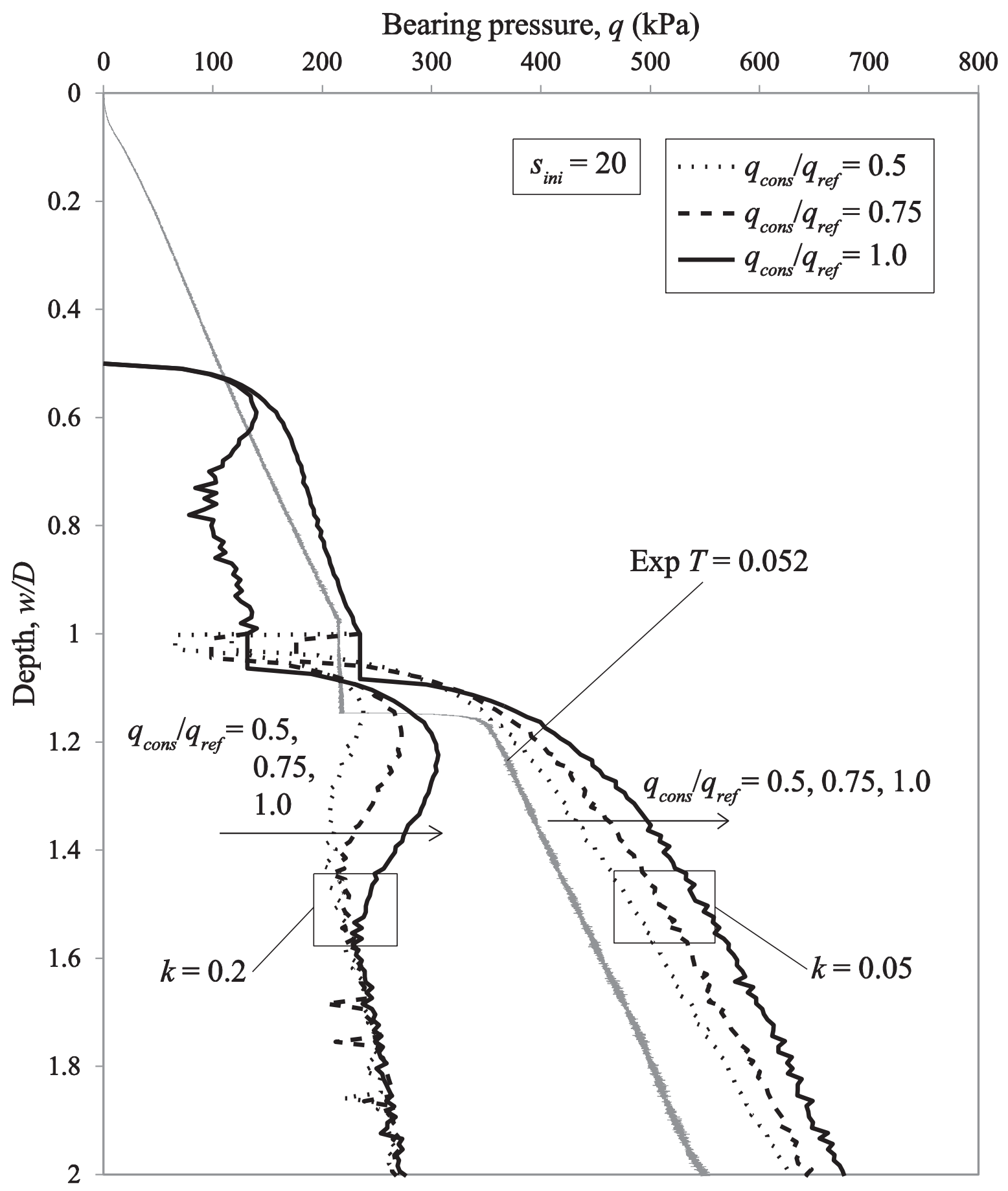

Figure 17: Effects of different load sustained in consolidation $q_{\text {cons }} / q_{\text {ref }}$ on the vertical bearing capacity profiles of natural soil with $s_{i n i}=20$. 

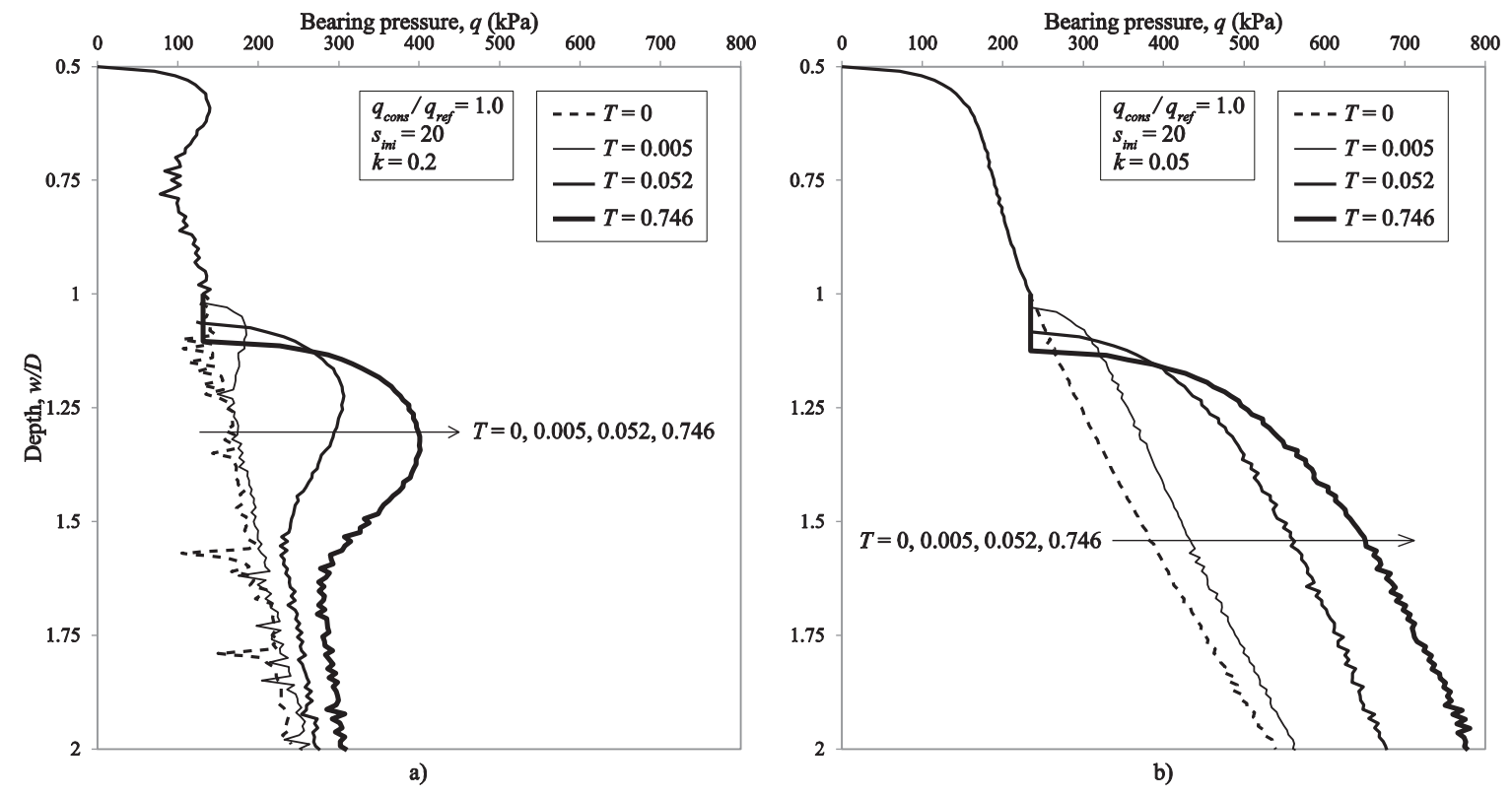

Figure 18: Effects of different consolidation length $T$ on the vertical bearing capacity profiles of natural soil with $s_{i n i}=20$. 